\title{
DINÁMICA DE LA CONCENTRACIÓN Y ACUMULACIÓN DE NUTRIMENTOS EN LOS COMPONENTES DE LA BIOMASA AÉREA DE Cedrela odorata L. EN COSTA RICA
}

\author{
Daniel Ramírez, Alfredo Alvarado ${ }^{1 / *}$, Carlos Ávila**, Manuel E. Camacho*, Jesús Fernández.*, \\ Rafael Murillo ${ }^{* *}$, Luis Salazar ${ }^{* * * *}$, Carlos L. Sandi ${ }^{* * * * * *}$ \\ Palabras clave: Cedro amargo; macro nutrimentos; micro elementos; nutrición mineral; neotrópico; Bosque \\ Muy Húmedo Tropical; Cedrela odorata. \\ Keywords: Spanish cedar; macro nutrients; micro elements; mineral nutrition; neotropics; Very humid tropical \\ forest; Cedrela odorata.
}

Recibido: $13 / 07 / 17$

Aceptado: 19/09/17

\section{RESUMEN}

Se planteó el presente estudio con el objetivo de estudiar la dinámica de la concentración y la acumulación de nutrimentos en árboles de Cedrela odorata L. (Meliaceae), para mejorar el conocimiento y la gestión de las plantaciones forestales tropicales. Mediante una serie falsa de tiempo (crono secuencia) compuesta por 15 plantaciones en bosque Muy Húmedo Tropical de Costa Rica, se determinó la biomasa aérea, la concentración de nutrimentos y se estimó su acumulación en los componentes de la biomasa en árboles seleccionados. Como resultado se obtuvo que la concentración de los nutrimentos presentó tendencia a disminuir en el orden: nitrógeno $>$ potasio $>$ magnesio $>$ calcio $>$ azufre $>$ fósforo para los macro nutrimentos, mientras que los micro elementos siguen la secuencia hierro $>$ boro $>$ zinc $>$ manganeso $>$ cobre $>$ aluminio.

\footnotetext{
1 Autor para correspondencia. Correo electrónico: alfredo.alvarado@ucr.ac.cr

Universidad de Costa Rica, Centro de Investigaciones Agronómicas, San José, Costa Rica.

** Universidad Nacional de Costa Rica, Instituto de Investigación y Servicios Forestales, Heredia, Costa Rica.
}

\begin{abstract}
Concentration dynamics and nutrient accumulation in above ground biomass components of Cedrela odorata L. in Costa Rica. Present study was conducted aiming to study nutrient concentration and accumulation dyna mics on Cedrela odorata L. (Meliaceae) trees to improve knowledge and management on tropical forestry plantations. False time series (chrono sequences) from 15 stands on Tropical Very Humid Forest of Costa Rica were used to determine aboveground biomass, nutrient concentration and to estimate nutrient accumulation on aboveground biomass components of selected trees. As a result, nutrient concentration showed a trend to decrease and followed the order nitrogen $>$ potassium $>$ magnesium $>$ calcium $>$ sulfur $>$ phosphorus for macro nutriments, while minor elements followed the order iron $>$ boron $>$ zinc

\footnotetext{
*** Bosques Naturales S.A. Alcobendas, Madrid, España.

**** CoopeAgri R.L. Pérez Zeledón, San José, Costa Rica.

***** Universidad EARTH, Las Mercedes de Guácimo, Limón, Costa Rica.
} 
Destacaron los altos valores de concentración de potasio medidos en los pecíolos $(1,46 \pm 0,48 \%)$ en contraste con contenidos más bajos de este elemento en el follaje $(1,09 \pm 0,21 \%)$. Se desarrolló modelos de regresión lineal para evaluar el comportamiento de la concentración de nutrimentos y se observó tendencia a la disminución de la concentración nitrógeno, fósforo, potasio y magnesio con el incremento de la edad, al ser este comportamiento más evidente en el potasio del fuste. Por otro lado, los modelos de regresión generados para evaluar el comportamiento de la acumulación de nutrimentos en los distintos componentes de la biomasa presentaron un comportamiento exponencial, con significancia estadística principalmente en el fuste y en el total acumulado. Dichos modelos permiten estimar posibles salidas de nutrimentos del sitio de plantación durante la cosecha, así como el reciclaje de cantidades de nutrimentos en componentes de la biomasa que permanecen en el sitio de plantación.

\section{INTRODUCCIÓN}

Cedrela odorata L. (Meliaceae), conocida como cedro amargo, es una especie arbórea de gran valor comercial originaria de México y Centroamérica, donde se distribuye en climas cálidos y semicálidos, asociada a los bosques tropicales húmedos y estacionales mixtos, semi-siempre verdes o semi-deciduos dominados por otras especies en las zonas de vida tropical y subtropical (Timyan 1996, Cavers et al. 2003). La especie se encuentra como individuos esparcidos y se adapta a las condiciones de suelos como Inceptisoles dístricos y Ultisoles (Alvarado 2012a). Su desarrollo en plantaciones se ha visto limitado por el ataque de Hypsipyla grandella y en Costa Rica es común encontrarla asociada a cultivos como café, pastos o cacao en la zona del Pacífico Sur y la vertiente Caribe.

Suministrar a los árboles los nutrimentos minerales de forma correcta es esencial para optimizar su desarrollo, hecho que en muchas plantaciones o bosques ocurre de manera suficiente de
$>$ manganese $>$ cooper $>$ aluminum. Distinguish high potassium concentration values on petioles were measured $(1.46 \pm 0.48 \%)$, contrasting lower values of this nutriment on leaves $(1.09 \pm 0.21 \%)$. Linear regression models were developed to evaluate behavior of nutrient concentration, and it was found a decreasing trend in nitrogen, phosphorus, potassium and magnesium concentration associated to tree age increase, been this behavior more evident on stem potassium concentration. On the other hand, regression models developed to evaluate behavior of nutrient accumulation on different aboveground biomass components presented exponential trend and statistical significance, especially on stem and total accumulation. These models allow to estimate possible nutrient outputs from the planting site during harvesting, as well as recycling of nutrient amounts into components of the biomass that remain at the planting site.

forma natural en el ecosistema y si se presentan deficiencias nutricionales, estas no suelen ser catastróficas ni muy obvias. Sin embargo, en plantaciones forestales el análisis foliar constituye una herramienta de diagnóstico para la predicción de elementos deficientes y posibles problemas de crecimiento (Dreschel y Zech 1991, Alvarado 2012b), así como un papel fundamental en estudios de acumulación y exportación de nutrimentos (Fernández et al. 2014, Camacho et al. 2016). Los estudios de acumulación de nutrimentos en especies forestales proveen información muy valiosa para el manejo sostenible de las plantaciones, dentro de la cual destacan modelos que permiten estimar las posibles salidas de nutrimentos del sitio de plantación como cosecha o el reciclaje de nutrimentos en los componentes de la biomasa aérea que permanecen en el sitio posterior a la cosecha, como las ramas y el follaje (Alvarado 2012b, Fernández et al. 2013, Camacho et al. 2017). 
Para $C$. odorata se conoce muy poco de la interacción de los diferentes componentes de la biomasa aérea y sus nutrimentos esenciales (Guevara 1988, Webb et al. 2000, Webb et al. 2001a,b, Paniagua 2005) o de otras propiedades del suelo sobre su crecimiento (Belanger y Briscoe 1963, Castaing 1982, Pérez 2009, Mateo et al. 2011, Hall et al. 2011, Rodríguez et al. 2015). Destaca el trabajo elaborado por Dreschel y Zech (1993) en el cual se definieron niveles de concentración foliar de 12 macro y micro elementos en especies tropicales, entre las que se encuentra la especie C. odorata. Sin embargo, en este último trabajo se limita a muestras foliares en árboles de 2 años para esta especie, por lo que se desconoce la dinámica de la concentración de nutrimentos a lo largo del ciclo de producción, tal y como se ha investigado en otras especies forestales en Costa Rica (Segura et al. 2006, Fernandez et al. 2014, Avellán et al. 2015, Camacho et al. 2016).

La presente investigación parte de la hipótesis de que la especie distribuye los distintos nutrimentos en cantidad y tipo de manera diferenciada entre sus componentes de la biomasa aérea en función de sus necesidades durante su período de crecimiento. Para comprobar el anterior planteamiento se plantea el objetivo de estudiar la dinámica de la concentración y acumulación de nutrimentos en los componentes de la biomasa aérea en plantaciones de $C$.odorata en monocultivo o en asocio con otros cultivos en sus diferentes etapas de desarrollo en el bosque muy húmedo de Costa Rica.

\section{MATERIALES Y MÉTODOS}

Sitios de muestreo: se realizaron muestreos en fincas de pequeños productores de árboles de cedro amargo (Cedrela odorata L.) escogidos entre los 70 proyectos homogéneos y de más de $1000 \mathrm{~m}^{2}$ de superficie asociados a CoopeAgri R.L. en la cuenca del río Grande de Térraba en San Isidro del General $(9,20123 \mathrm{~N}$, $-83,59311 \mathrm{O} ; 9,38809 \mathrm{~N},-83,66338 \mathrm{O})$ y otros en la finca experimental de la Escuela Agrícola Regional del Trópico Húmedo (EARTH) en las llanuras caribeñas de Costa Rica $(10,18494 \mathrm{~N}$ y -83,60426 O) (Figura 1). Ambas localidades se encuentran dentro de la zona de vida del bosque muy húmedo premontano, donde las lluvias oscilan desde los 2000 y $4000 \mathrm{~mm}$ anuales, la temperatura promedio anual oscila entre 18 y $26^{\circ} \mathrm{C}$ (Bolaños et al. 2005) y los suelos que clasifican como Ultisoles asociados con Inceptisoles. En la región de San Isidro del General varios de los sitios escogidos se encontraban intercalados en sistemas agroforestales con pastos y plantaciones de café y cacao (árboles de 4, 7 y 8 años), mientras que los demás sitios de estudio se encontraban en plantaciones puras. 


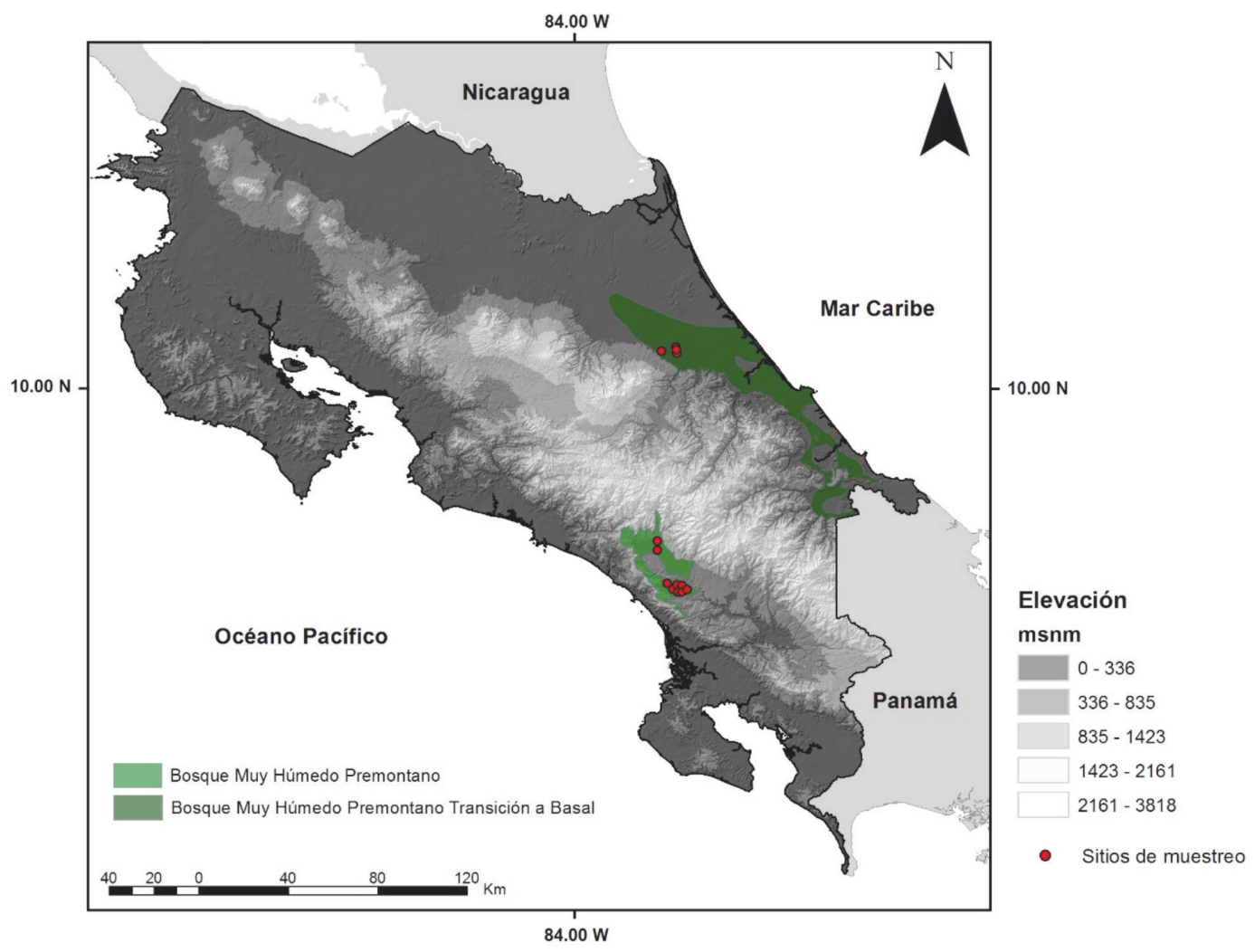

Fig. 1. Áreas de muestreo de árboles de cedro amargo (Cedrela odorata L.) en las regiones Pacífico Sur y Caribe de Costa Rica.

Escogencia de los árboles de muestreo: se utilizó el método de series de tiempo falsas para la determinación del comportamiento en la acumulación y variación de nutrimentos en árboles de C. odorata de edades entre 2 y 19 años. Al seguir los criterios empleados por Castaing (1982) se muestrearon individuos de crecimiento y desarrollo promedio en comparación a los demás árboles del sitio durante la época lluviosa, de manera que el nivel de competencia por espacio y luz fuera homogéneo, sin ningún problema fitosanitario, sin bifurcación en el tronco, ni árboles de los bordes de caminos o de las parcelas muestreadas. Los árboles escogidos se cortaron lo más cercano posible al suelo después de medir su diámetro a altura de pecho (DAP), para luego medir su altura total y separar sus componentes de la biomasa aérea agrupándolos en ramas primarias y secundarias, tronco (fuste), hojas y pecíolos (raquis y pecíolos). En el campo se determinó la densidad de siembra utilizada por los parceleros, la cual varió en función del sistema de plantación en asocio con varios cultivos.

\section{Muestreo de los componentes de la bio-} masa aérea: cada componente de la biomasa aérea se tomó por aparte y se colocó la muestra en una bolsa con información sobre la edad de los árboles y de la plantación, lote, lugar, fecha, parte del árbol y sitio. El tronco con corteza de cada árbol fue dividido en secciones y cada una de estas se pesó y se anotó su peso al tomarse 
muestras de las secciones impares, las cuales se colocaron sobre un plástico y se cortaron con una motosierra para obtener una muestra del aserrín homogeneizado que se colocó en una bolsa plástica hermética. Las ramas primarias y secundarias se separaron del tronco principal, se colocaron sobre un plástico para evitar que se contaminaran con suelo, se pesaron en una balanza y se anotó su peso; las muestras de aserrín de este componente se hicieron al cortar las ramas con una motosierra, al eliminar las basuras y cualquier tipo de residuo contaminante. Las muestras de follaje se tomaron al recolectar la totalidad de hojas de cada árbol sobre una lona o plástico de polietileno, se las separó del raquis, (con el pecíolo) que se muestreó por aparte, se pesaron en una balanza, se anotó el peso fresco y se tomó una muestra de 40 hojas y de todos los pecíolos, las cuales se colocaron en bolsas plásticas debidamente rotuladas para su posterior análisis.

Análisis químico de los componentes: los componentes de la biomasa se pesaron y de ellos se tomaron submuestras que fueron analizadas en el Laboratorio de Suelos y Foliares del Centro de Investigaciones Agronómicas de la Universidad de Costa Rica (CIA-UCR). Las muestras frescas de los diferentes componentes de la biomasa aérea se colocaron en una estufa a $70^{\circ} \mathrm{C}$ por un período de 48 horas para determinar el peso seco con una balanza digital con precisión de $0,01 \mathrm{~g}$. Con base en el peso seco y peso fresco se determinó el porcentaje de humedad de los diferentes componentes y se calculó el peso seco total para cada uno. Las muestras fueron posteriormente digeridas con ácido nítrico y en la solución resultante se determinó con espectrometría de emisión atómica con plasma acoplado (ICP) el contenido de potasio, calcio, magnesio, azufre, hierro, cobre, zinc, manganeso, boro y aluminio (Kalra 1998) y el fósforo por fotometría siguiendo el método del azul de molibdeno (Murphy y Riley 1962). La concentración de nitrógeno se obtuvo mediante combustión seca en un analizador de carbono, hidrógeno, oxígeno y nitrógeno por el método de Dumas en un auto-analizador con incineración a $500^{\circ} \mathrm{C}$. Con la información de laboratorio se calculó la cantidad absoluta de nutrimentos absorbidos como la sumatoria de porcentajes de todos los elementos en cada especie.

Análisis de datos: con los datos de humedad de las muestras se determinó la biomasa seca de cada componente de la biomasa aérea para cada árbol muestreado. Se ajustó un modelo de regresión de forma potencial para medir la biomasa del fuste, ramas primarias y secundarias, pecíolos y follaje de acuerdo con la siguiente ecuación:

$$
y=a x^{b}
$$

Donde la variable independiente $(x)$ representa la edad del árbol en años y la biomasa aérea total en kg.árbol ${ }^{-1}$ es la variable dependiente en la ecuación 1. Para estos modelos se evaluó el $\mathrm{R}^{2}$ ajustado linearizando el modelo exponencial, la probabilidad del modelo $(p)$ y de los parámetros de regresión $(a)$ y $(b)$, así como pruebas de normalidad de los datos y pruebas de varianza de constantes.

Los resultados de concentración de cada nutrimento de las muestras analizadas en base al peso seco se ingresaron a una base de datos. Por elemento y por componente de la biomasa aérea se estimó el valor promedio y el error estándar. Se ajustaron modelos de regresión de forma lineal para la concentración de cada elemento por cada componente de la biomasa aérea del árbol, de acuerdo con la siguiente ecuación:

$$
y=\beta_{1}(x)+\beta_{0}
$$

Donde la variable independiente $(x)$ corresponde a la edad del árbol (años) y la concentración (\%) de nitrógeno, fósforo, potasio, calcio, magnesio, azufre y en mg. $\mathrm{kg}^{-1}$ para hierro, cobre, zinc, manganeso, boro y aluminio como variables dependientes $(y)$ en la ecuación 2. Para los modelos anteriores se evaluó el $\mathrm{R}^{2}$, la probabilidad $p$ del modelo y de los parámetros de regresión $\beta_{l} \mathrm{y}$ $\beta_{0}$, así como pruebas de normalidad de los datos y pruebas de varianza constante. 
Se calculó la acumulación de nutrimentos por componente multiplicando la biomasa seca por la concentración, obtenidos anteriormente de acuerdo con las siguientes fórmulas:

Para los macro elementos:

kg.nutrimento componente $e^{-1}=$ biomasa seca del componente $(\mathrm{kg}) * \frac{\text { nutrimento }(\%)}{100}$

Para los elementos menores:

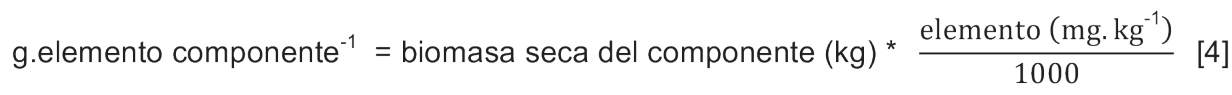

Con los valores de acumulación de nutrimentos obtenidos en las ecuaciones 3 y 4 se ajustaron modelos de regresión de forma exponencial para cada componente de la biomasa aérea del árbol:

$$
y=a b^{x}
$$

Donde la edad del árbol (años) es la variable independiente $(x)$ y el valor de acumulación es la variable dependiente $(y)$ en la ecuación 5 , en kg.árbol ${ }^{-1}$ para los macro nutrimentos y g.árbol ${ }^{-1}$ para los micro elementos. Para los modelos anteriores se evaluó el $\mathrm{R}^{2}$ alineado a un modelo exponencial, su probabilidad $(p)$ y los parámetros de regresión $(a)$ y $(b)$, así como pruebas de normalidad de los datos y pruebas de varianza de constantes. Los modelos desarrollados tanto para la acumulación de biomasa, concentración y acumulación de nutrimentos, se generaron mediante el uso de los paquetes informáticos Sigmaplot ${ }^{\circledR}$ e InfoStat $^{\circledR}$ (Systat Software, Inc. 2008, DiRienzo et al. 2012).

\section{RESULTADOS}

\section{Producción de biomasa aérea seca en los componentes de $C$. odorata}

Se determinó el crecimiento de los árboles de C. odorata con edades entre 1 y 19 años, expresado como acumulación de biomasa seca en las hojas, pecíolos, ramas primarias y secundarias, fuste y total, mediante una serie de tiempo falsa. La acumulación de biomasa seca de hojas, pecíolos y ramas (biomasa de copa) presentó comportamientos similares entre sí, pero diferentes al obtenido para la biomasa seca del fuste y total (Cuadro 1). Para los 3 primeros componentes, la biomasa tiende a crecer lentamente y se estabiliza una vez que se forma la copa; la autopoda y la dehiscencia natural o inducida por plagas, durante el período de crecimiento estudiado, permiten explicar el comportamiento de los datos. Se observó un incremento exponencial en la biomasa aérea total y del fuste asociado al aumento en la edad del árbol. Por lo anterior, las relaciones H/F y $(\mathrm{H}+\mathrm{P}) / \mathrm{F}$ disminuyen hasta la edad de 3 años como resultado del crecimiento natural de la especie y a una mayor competencia intraespecífica causada por el crecimiento de las ramas que se refleja en la relación R/F (Cuadro 1). En general, la distribución en la acumulación de biomasa seca en los distintos componentes presentó el siguiente el orden:

Fuste $(\mathrm{F})>$ Ramas $(\mathrm{R})>$ Hojas $(\mathrm{H})>$ Pecíolos $(\mathrm{P})$ 
Cuadro 1. Valor promedio de la biomasa seca de los componentes de árboles de $C$. odorata de 1 a 19 años estudiados en el Trópico Muy Húmedo de Costa Rica.

\begin{tabular}{ccccccccc}
\hline Edad & $\begin{array}{c}\text { Hojas } \\
(\mathrm{H})\end{array}$ & $\begin{array}{c}\text { Pecíolos } \\
(\mathrm{P})\end{array}$ & $\begin{array}{c}\text { Ramas } \\
(\mathrm{R})\end{array}$ & $\begin{array}{c}\text { Fuste } \\
(\mathrm{F})\end{array}$ & $\begin{array}{c}\text { Total } \\
(\mathrm{T})\end{array}$ & $\mathrm{H} / \mathrm{F}$ & $\mathrm{R} / \mathrm{F}$ & $(\mathrm{H}+\mathrm{P}) /(\mathrm{F}+\mathrm{R})$ \\
\hline Años & & & $\mathrm{kg}$. rbol $^{-1}$ & & & & & \\
\hline 1 & 0,1 & 0,1 & 0,0 & 0,3 & 0,5 & 0,41 & 0,00 & 0,77 \\
3 & 1,3 & 0,4 & 1,5 & 5,7 & 8,9 & 0,23 & 0,26 & 0,24 \\
4 & 1,0 & 0,3 & 23,0 & 38,3 & 62,6 & 0,03 & 0,60 & 0,02 \\
5 & 4,3 & 0,9 & 29,8 & 60,8 & 95,8 & 0,07 & 0,49 & 0,06 \\
7 & 2,7 & 0,1 & 17,2 & 45,6 & 65,7 & 0,06 & 0,38 & 0,05 \\
8 & 4,4 & 1,6 & 62,3 & 62,9 & 131,2 & 0,07 & 0,99 & 0,05 \\
9 & 6,4 & 1,9 & 36,2 & 86,0 & 130,5 & 0,07 & 0,42 & 0,07 \\
10 & 7,3 & 4,0 & 70,7 & 122,8 & 204,8 & 0,06 & 0,58 & 0,06 \\
14 & 7,7 & 3,0 & 22,0 & 320,1 & 352,8 & 0,02 & 0,07 & 0,03 \\
19 & 5,7 & 2,1 & 6,9 & 378,5 & 393,2 & 0,01 & 0,02 & 0,02 \\
\hline
\end{tabular}

El modelo de regresión obtenido para la estimación de la biomasa aérea total (Figura 2) presentó un buen ajuste y significancia estadística $\left(\mathrm{R}^{2}=0,94 ; \mathrm{p}<0,001\right)$, por lo que se considera satisfactorio en la estimación de la biomasa seca total en plantaciones de C. odorata en el ecosistema bosque tropical muy húmedo. Se generaron otros modelos similares (datos no presentados) para medir la biomasa seca del follaje y ramas, sin embargo, los valores de probabilidad (p-valor) tanto de los parámetros de ajuste como el de los modelos, no fueron estadísticamente significativos $(\mathrm{p}>0,05)$ anudado a bajos valores de $\mathrm{R}^{2}$. 


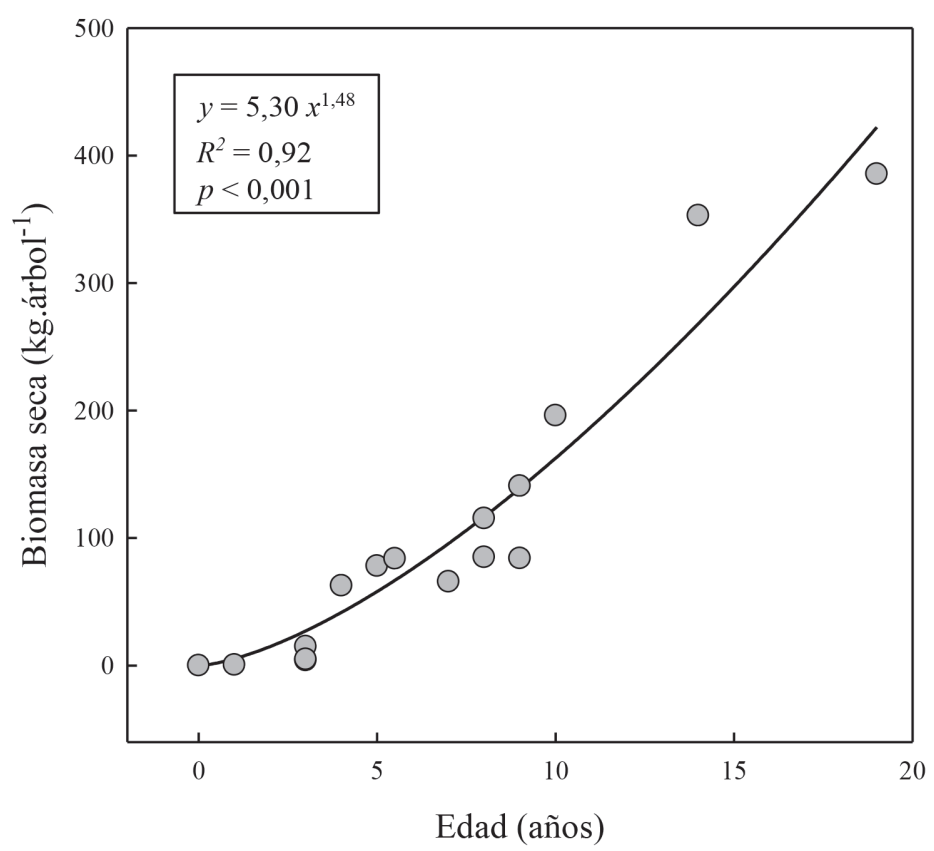

Fig. 2. Acumulación de biomasa aérea total seca en árboles de C. odorata en el Trópico Muy Húmedo de Costa Rica. Línea en negro representa el modelo de regresión de mejor ajuste. Modelo de mejor ajuste de forma potencial $y=a x^{b}$. Para la estimación del $\mathrm{R}^{2}$ se linearizó en modelo exponencial.

Con base en el comportamiento de la biomasa evaluada en los distintos componentes, se observó que a partir del momento de la plantación en el campo, la especie presenta 3 etapas de crecimiento: 1) desde la edad de 0 a 3 años, cuando la producción de biomasa aérea del árbol crece de forma lenta y depende de la fertilidad natural del sitio o de los nutrimentos agregados como fertilizante a los cultivos asociados; 2) de los 4 y hasta alrededor de los 9 años, cuando la biomasa de fuste y ramas crece de forma moderada, se inicia la pérdida natural de sus hojas a partir del quinto año y en plantaciones la biomasa total se ve afectada por las podas y raleos; y 3) a partir de los 10 y hasta los 19 años, cuando la biomasa aumenta hasta alcanzar alrededor de los $400 \mathrm{~kg}$ por árbol con incrementos diamétricos importantes con el paso de los años.

\section{Concentración de nutrimentos en los componentes de la biomasa aérea de $C$. odorata}

Se encontró variación en la concentración de los nutrimentos asociada a los distintos componentes de la biomasa aérea en árboles de $C$. Odorata, la que tiende a disminuir en función del componente de la biomasa, de acuerdo con el siguiente orden:

$$
\text { Hojas }>\text { Pecíolos }>\text { Ramas }>\text { Fuste }
$$

Los valores promedio de concentración para cada componente de la biomasa aérea se presentan en las Figuras 3 y 4. Las barras de error de los promedios fueron relativamente pequeñas, lo que sugiere posibles diferencias significativas entre las concentraciones en los 
componentes del árbol (Figuras 3). Los valores más elevados en la concentración de nitrógeno $(3,33-2,45 \%)$ se obtuvieron en las hojas, mientras que los valores más bajos $(0,90-0,36 \%)$ se presentaron en el fuste (Figura 3). Este comportamiento se presentó en otros macro nutrimentos como el fósforo $(0,23-0,18$ y $0,08-0,02 \%)$, calcio $(1,45-0,50$ y $0,57-0,20 \%)$, magnesio $(0,24-0,12$ y $0,06-0,03 \%)$ y azufre $(0,28-0,17$ y $0,07-0,02 \%)$. El potasio presentó sus valores más elevados en los peciolos $(2,35-1,17 \%)$, mientras que los valores más bajos se observaron en el fuste $(0,66-0,20 \%)$.
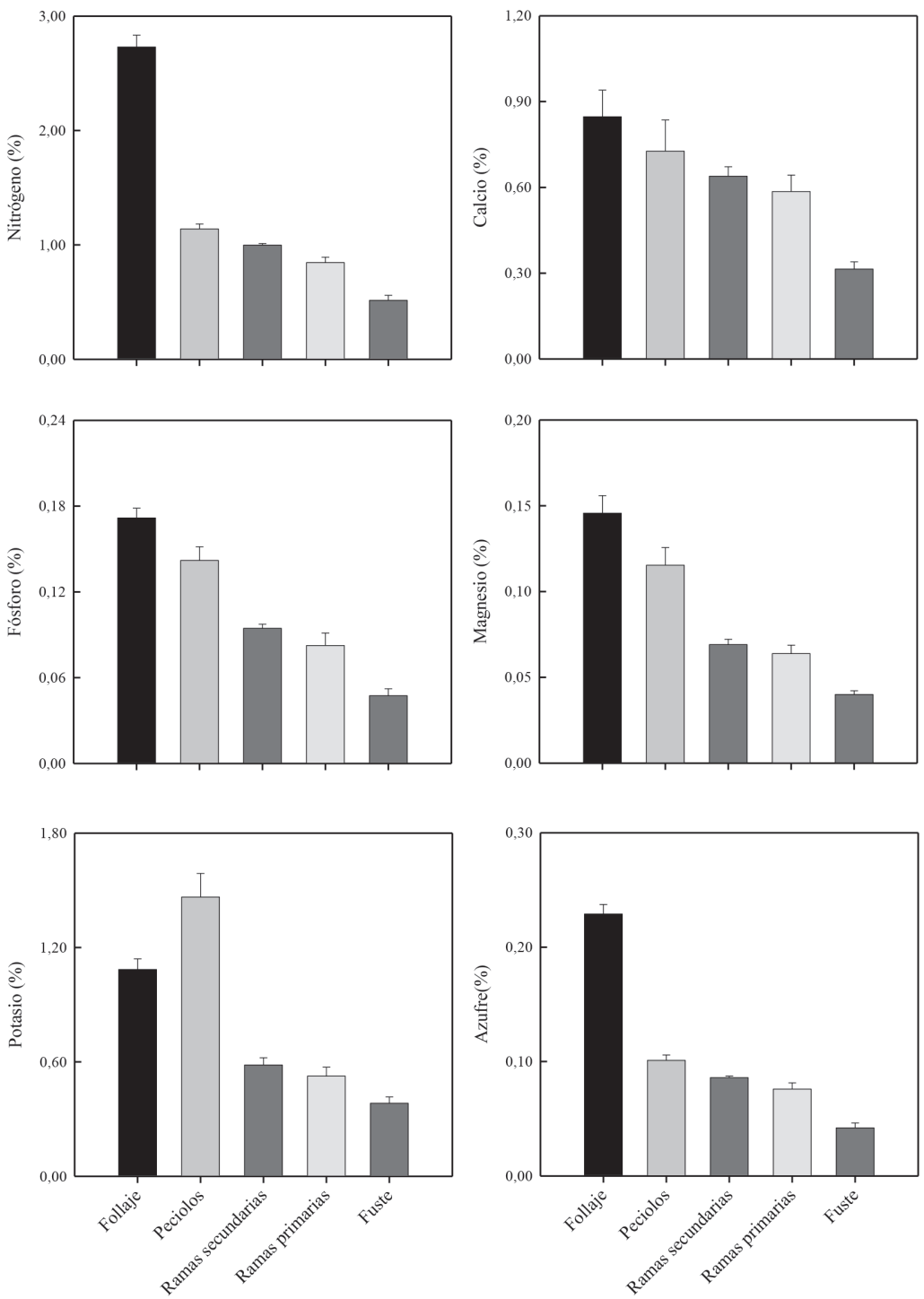

Fig. 3. Valores promedio de la concentración de macro nutrimentos (\%) en los distintos componentes de la biomasa aérea de árboles de $C$. odorata de edades entre 1 y 19 años del Trópico Muy Húmedo de Costa Rica. Barras de error como error estandar. 

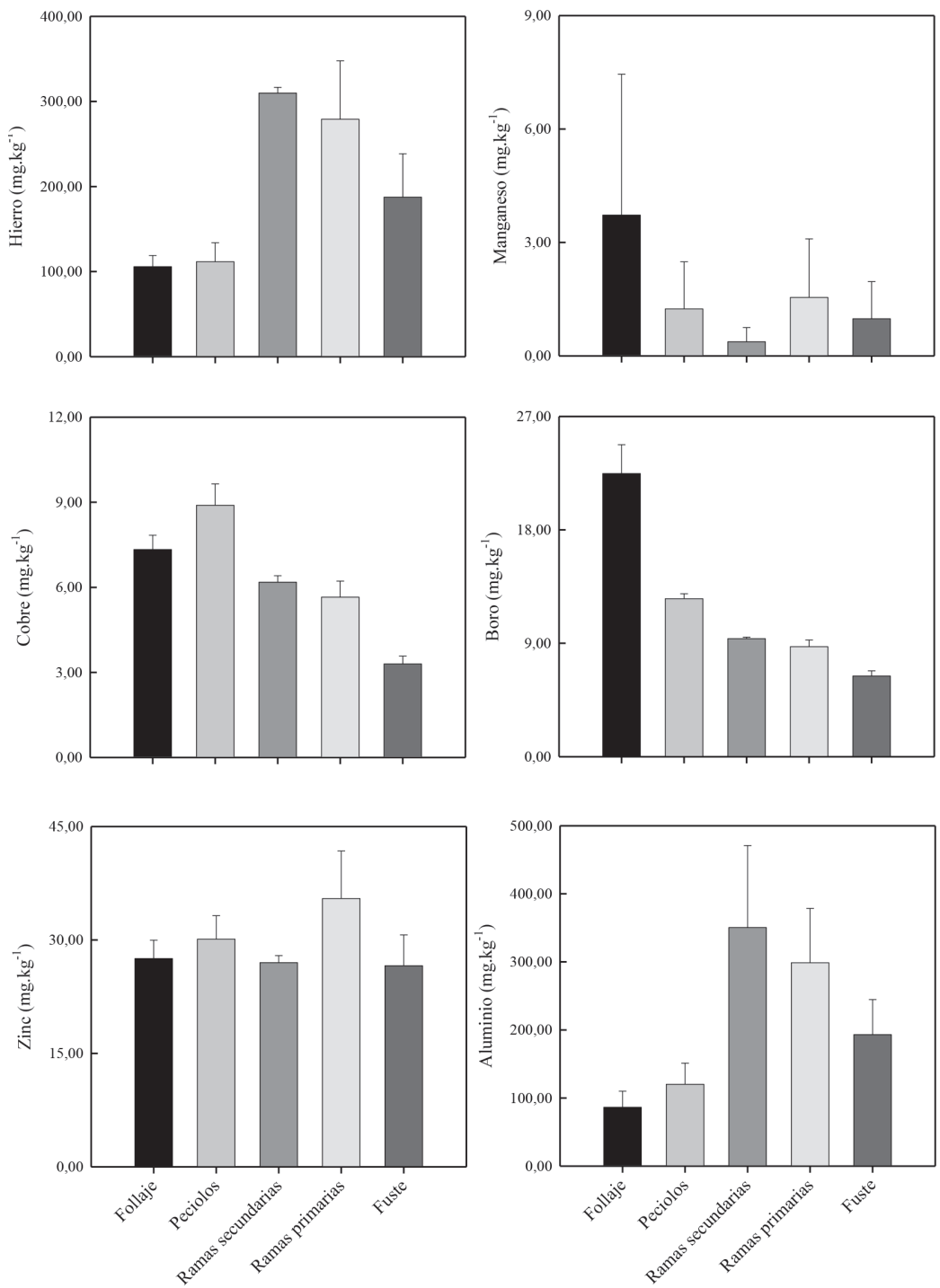

Fig. 4. Valores promedio de la concentración $\left(\mathrm{mg}_{\mathrm{kg}} \mathrm{kg}^{-1}\right)$ de micro elementos en los distintos componentes de la biomasa aérea de árboles de $C$. odorata de edades entre 1 y 19 años del Trópico Muy Húmedo de Costa Rica. Barras de error como error estandar.

En el caso de los microelementos destacan los valores más elevados de concentración para el boro (14-48 mg.kg-1) en las hojas, en cambio los valores más bajos $\left(5-10 \mathrm{mg} \cdot \mathrm{kg}^{-1}\right)$ se presentaron en el fuste (Figura 4). La concentración de aluminio presentó sus valores más elevados en las ramas primarias (40-1417 mg.kg-1), mientras que los valores más bajos se obtuvieron en las hojas (22-385 mg.kg-1 ${ }^{-1}$. Se observó que la concentración de los macro nutrimentos y micro elementos presentó tendencias en su variación asociadas a cada componente de la biomasa aérea de la siguiente manera: 
Hojas: Nitrógeno $>$ Potasio $>$ Calcio $>$ Azufre $>$ Magnesio $>$ Fósforo Hierro $>$ Zinc $>$ Aluminio $>$ Boro $>$ Manganeso $>$ Cobre Pecíolos:Nitrógeno $>$ Magnesio $>$ Potasio $>$ Calcio $>$ Azufre $>$ Fósforo Boro $>$ Hierro $>>$ Cobre $>$ Zinc $>$ Manganeso $>$ Aluminio

Ramas Nitrógeno $>$ Potasio $>$ Magnesio $>$ Calcio $>$ Azufre $=$ Fósforo Hierro $=$ Boro $>$ Cobre $>$ Zinc $>$ Manganeso $>$ Aluminio

Fuste: Nitrógeno $>$ Potasio $>$ Calcio $>$ Azufre $=$ Fósforo $=$ Magnesio Aluminio $>$ Hierro $>>$ Zinc $>$ Manganeso $=$ Boro $=$ Cobre

Al evaluar la variación en la concentración de nutrimentos en función de la edad de los árboles se encontró que la concentración de nitrógeno, potasio y magnesio en el fuste, así como potasio y fósforo en el follaje, tienden a disminuir al aumentar la edad (Figura 5), este comportamiento es más evidente en el potasio del fuste. Los modelos de regresión lineal desarrollados para evaluar esta interacción entre la edad del árbol y la concentración de los elementos, presentaron significancia estadística con un ajuste de modelo muy bajo $\left(\mathrm{R}^{2}<0,30\right)$, con excepción de la concentración del potasio en el fuste, cuyo modelo presentó un valor de $\mathrm{R}^{2}=0,68$ (Cuadro 2; Figura 5). Al considerar la variación de los nutrimentos del árbol de forma integral, se observó que esta disminuye para los macro nutrimentos en el orden nitrógeno $>$ potasio $>$ magnesio $>$ calcio $>$ azufre $>$ fósforo y para los micro elementos en la secuencia hierro $>$ boro $>$ zinc $>$ manganeso $>$ cobre $>$ aluminio. 

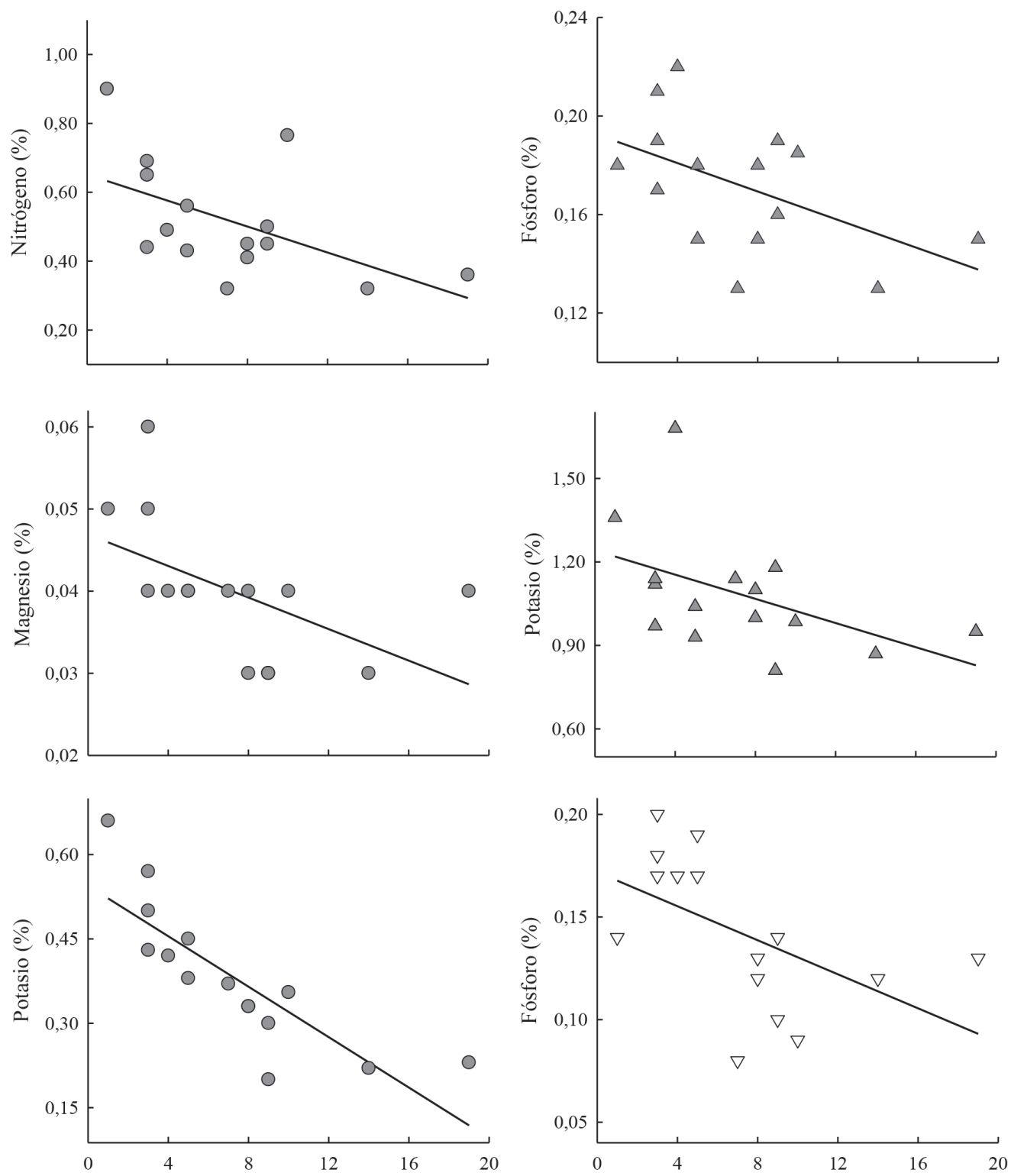

Edad (años)

\begin{tabular}{|lll|}
\hline$\bigcirc$ & Fuste & $\nabla \quad$ Peciolos \\
$\triangle$ & Follaje & \\
\cline { 2 - 2 }
\end{tabular}

Fig. 5. Variación de la concentración de nitrógeno, fósforo, potasio y magnesio con la edad en componentes selectos de la biomasa aérea de árboles de $C$. odorata del Trópico Muy Húmedo de Costa Rica. Los modelos de mejor ajuste se detallan en el Cuadro 2. 


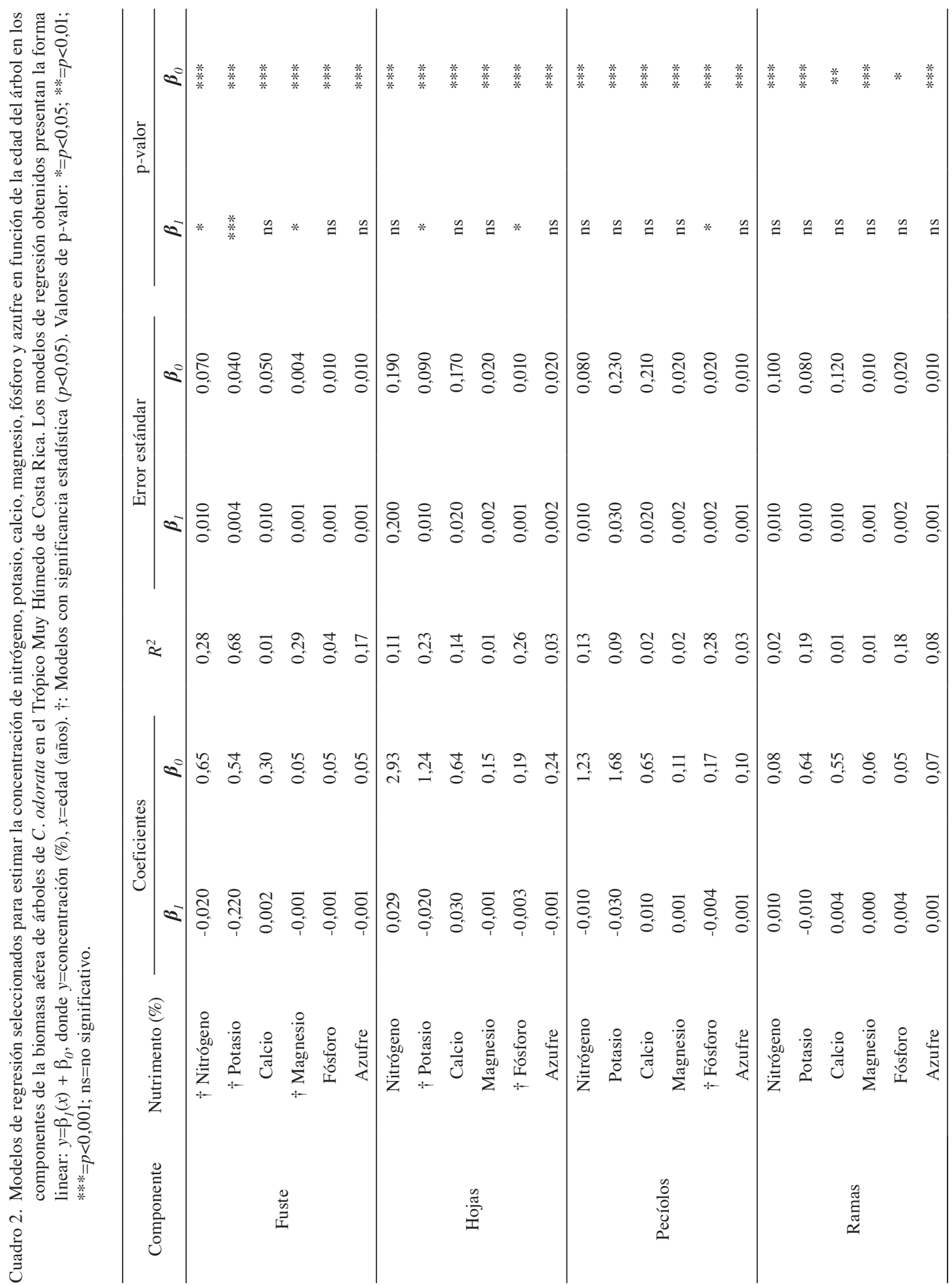


Acumulación de nutrimentos en los componentes de la biomasa aérea de $C$. odorata

Los modelos propuestos para la estimación de la acumulación de nutrimentos se presentan en los Cuadros 3 y 4 . De forma general, se observó un incremento en la acumulación de nutrimentos asociado a la edad del árbol, principalmente en el fuste, en el follaje y en el total acumulado; este último influenciado por el comportamiento en la acumulación de biomasa seca en el fuste y por ende en el total acumulado por el árbol. 


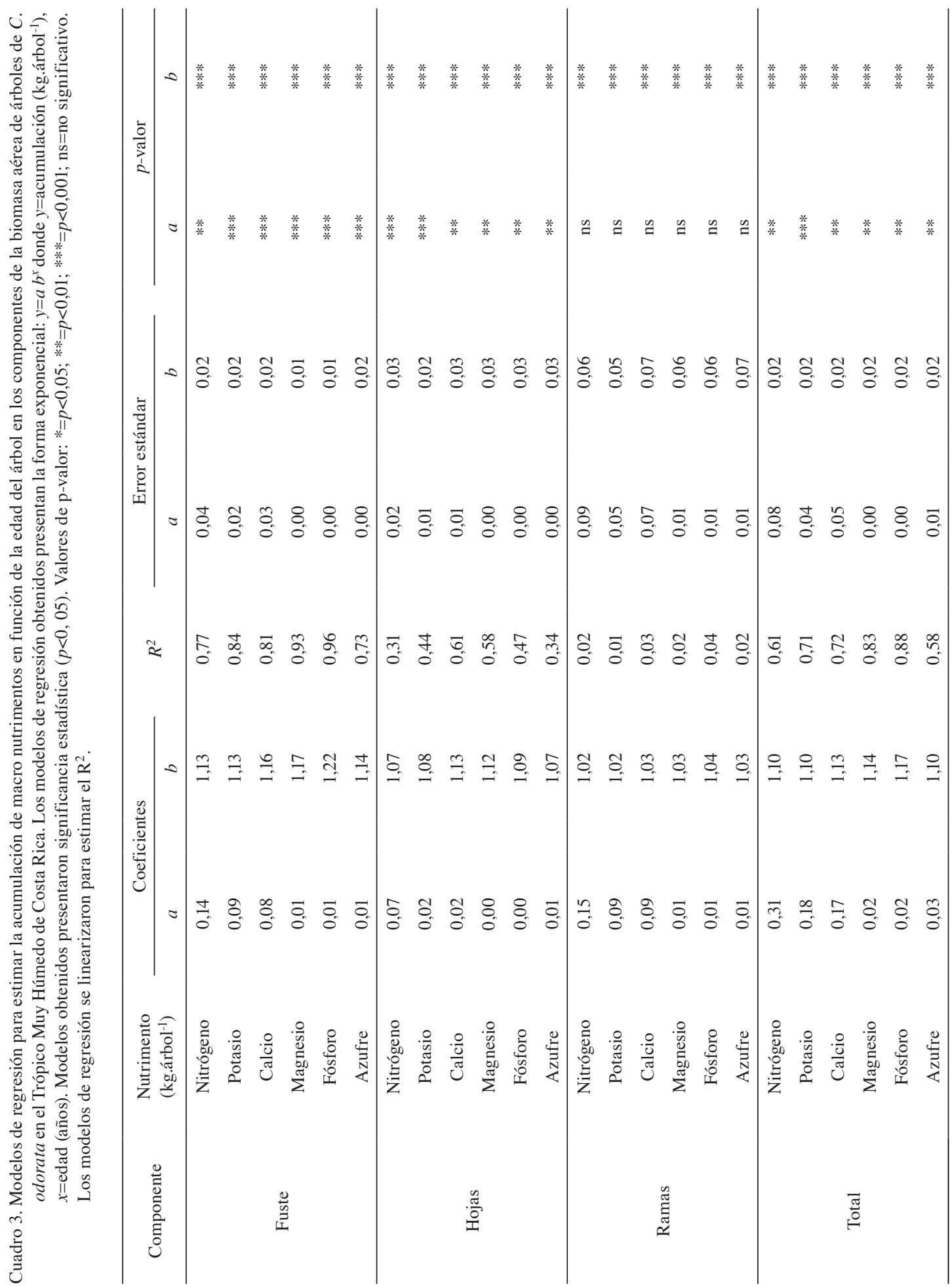




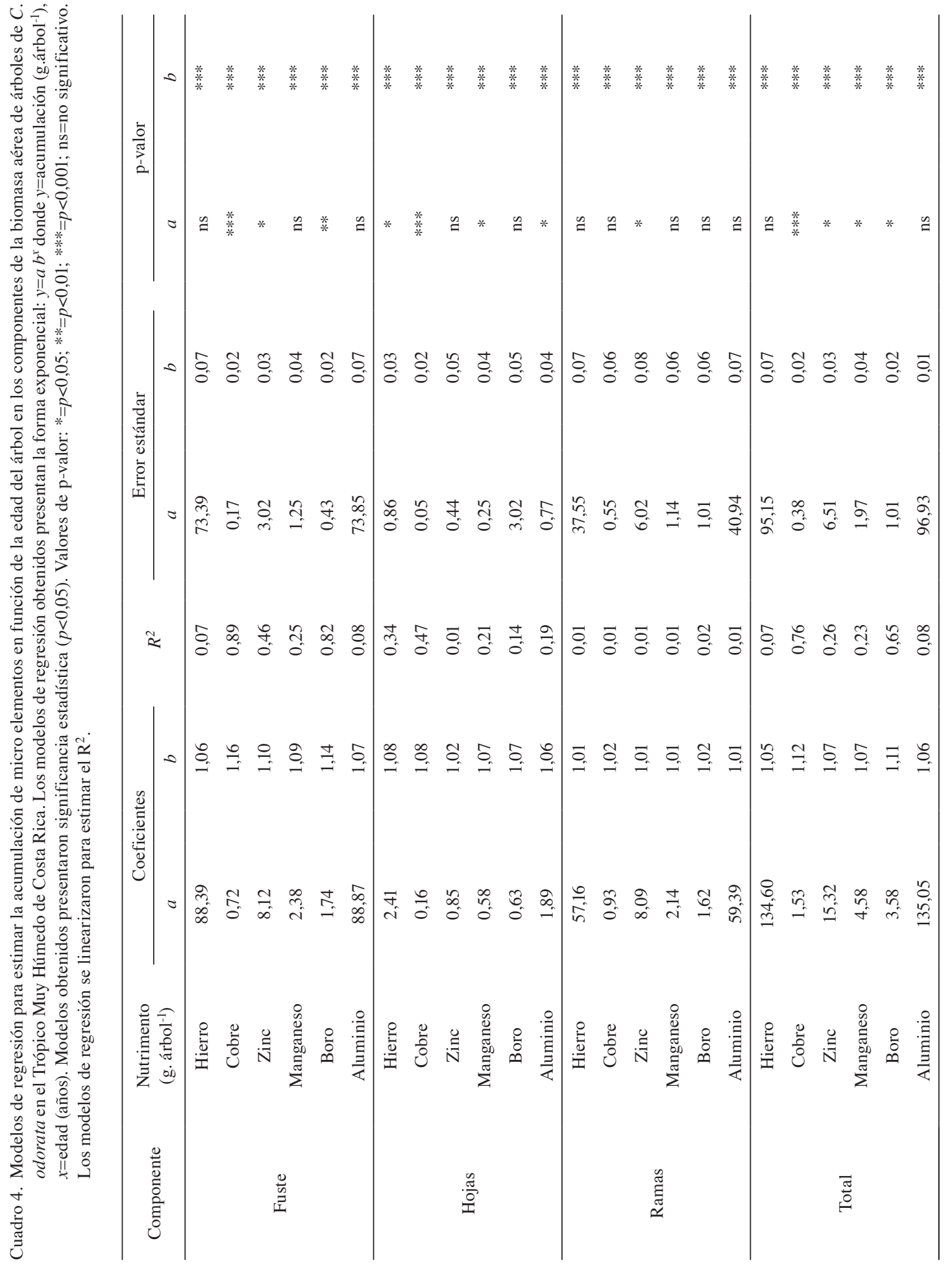


Los valores máximos de acumulación de nitrógeno en los componentes de la biomasa aérea en los árboles evaluados (Figura 6) se encontraron en el fuste (1,36 kg.árbol-1), seguido de las ramas y follaje $(0,69$ y 0,24 $\mathrm{kg}$.árbol-1 ${ }^{-1}$. Los modelos de mejor ajuste obtenidos para la acumulación de nitrógeno total y en el fuste presentaron $R^{2}$ elevados $\left(R^{2}>0,75\right)$ y significancia estadística (Cuadro 3). Los modelos desarrollados para medir la acumulación de nitrógeno en los restantes componentes (ramas y follaje) también presentaron significancia estadística (Cuadro 3), pero sus valores de $R^{2}(<0,30)$ fueron bajos, probablemente por el comportamiento de la biomasa en ambos componentes.
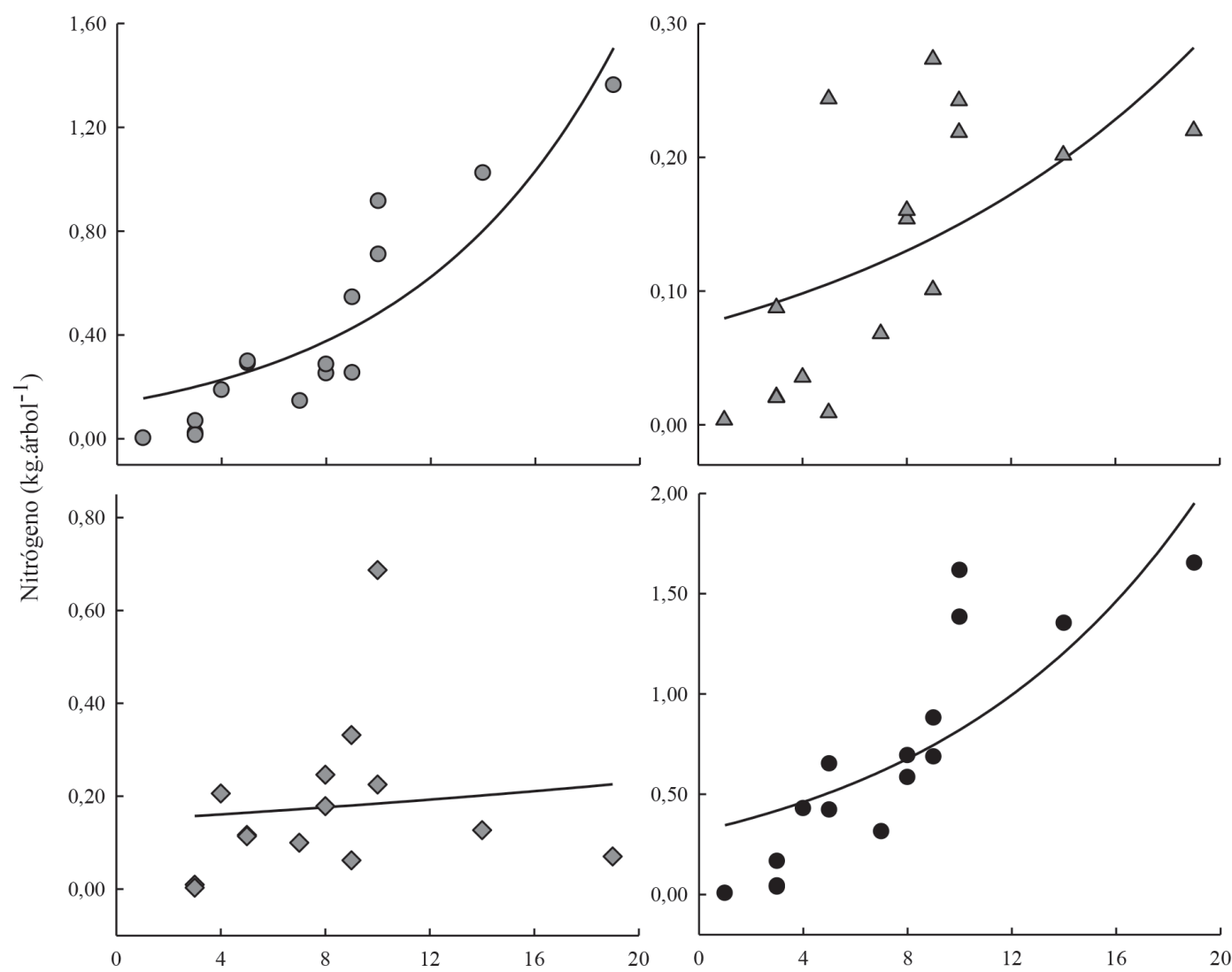

Edad (años)

\begin{tabular}{|lllll}
\hline & Fuste & $\Delta$ & Follaje \\
$\diamond$ & Ramas & Total
\end{tabular}

Fig. 6. Acumulación de nitrógeno en función de la edad, en distintos componentes de la biomasa aérea de árboles de $C$. odorata en el Trópico Muy Húmedo de Costa Rica. Los modelos de mejor ajuste se detallan en el Cuadro 3. 
La acumulación de calcio, fósforo y potasio presentó comportamientos similares al nitrógeno, es decir, valores máximos de acumulación en el fuste $\left(1,32 ; 0,87\right.$ y $0,26 \mathrm{~kg}$.árbol $\left.^{-1}\right)$ y los valores más bajos de acumulación $(0,11 ; 0,02$ y $0,26 \mathrm{~kg}$.árbol ${ }^{-1}$ ) en el follaje (Figuras 7, 8 y
9). Los modelos de mejor ajuste para medir la acumulación total y en el fuste de estos 3 nutrimentos, presentaron significancia estadística y $\mathrm{R}^{2}$ elevados, mientras que en los modelos propuestos en las ramas y el follaje presentaron significancia estadística y valores de $\mathrm{R}^{2}$ muy bajos (Cuadro 3).
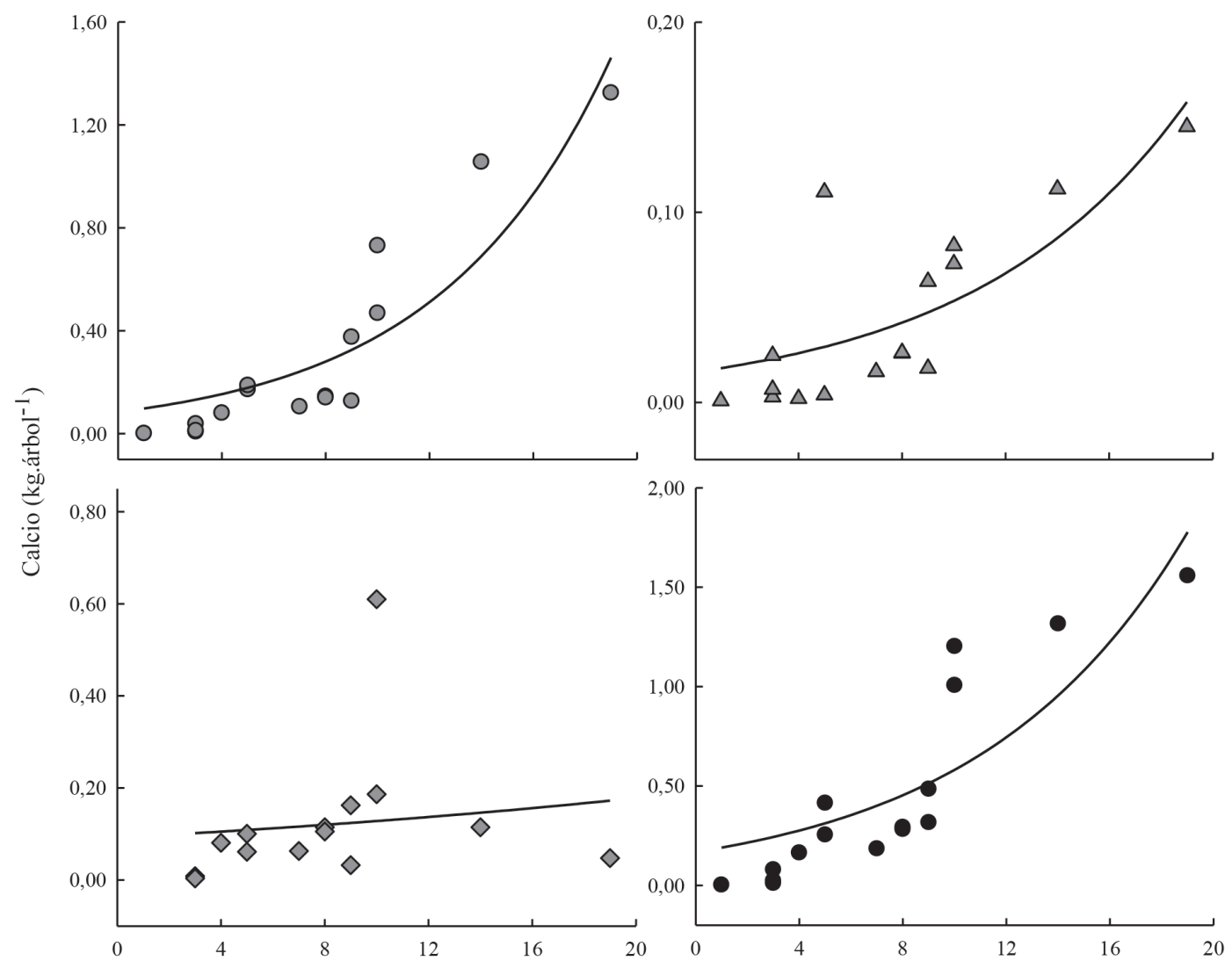

Edad (años)

\begin{tabular}{|cllll|}
\hline$\diamond$ & Fuste & $\Delta$ & Follaje & \\
$\diamond$ & Ramas & $\bullet$ & Total & \\
\hline
\end{tabular}

Fig. 7. Acumulación de calcio en función de la edad en los distintos componentes de la biomasa aérea de árboles de $C$. odorata en el Trópico Muy Húmedo de Costa Rica. Los modelos de mejor ajuste se detallan en el Cuadro 3. 

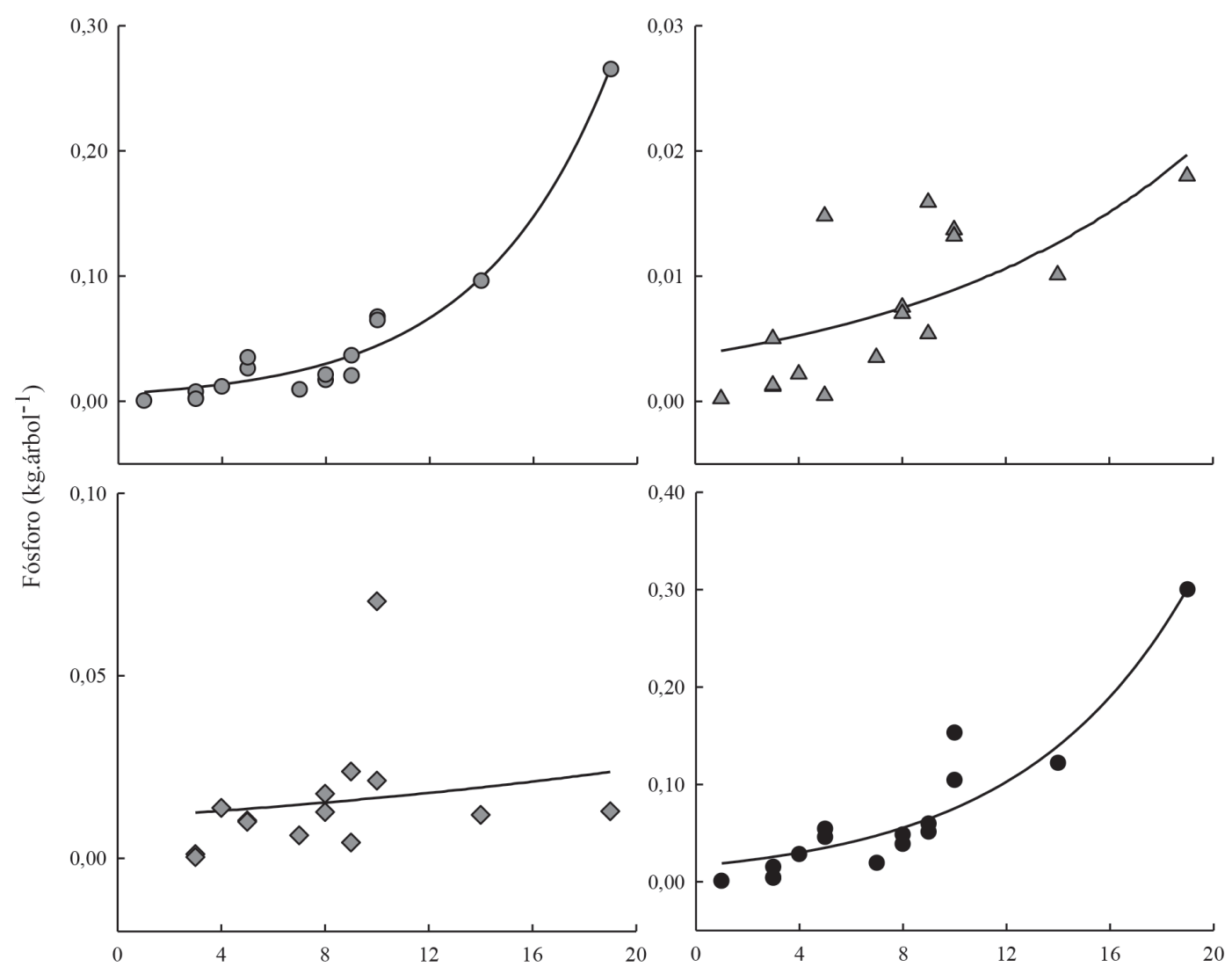

Edad (años)

\begin{tabular}{|ccccc|}
\hline$\bigcirc$ & Fuste & $\Delta$ & Follaje & \\
$\diamond$ & Ramas & $\bullet$ & Total & \\
\hline
\end{tabular}

Fig. 8. Acumulación de fósforo en función de la edad en los distintos componentes de la biomasa aérea de árboles de $C$. odorata en el Trópico Muy Húmedo de Costa Rica. Los modelos de mejor ajuste se detallan en el Cuadro 3. 


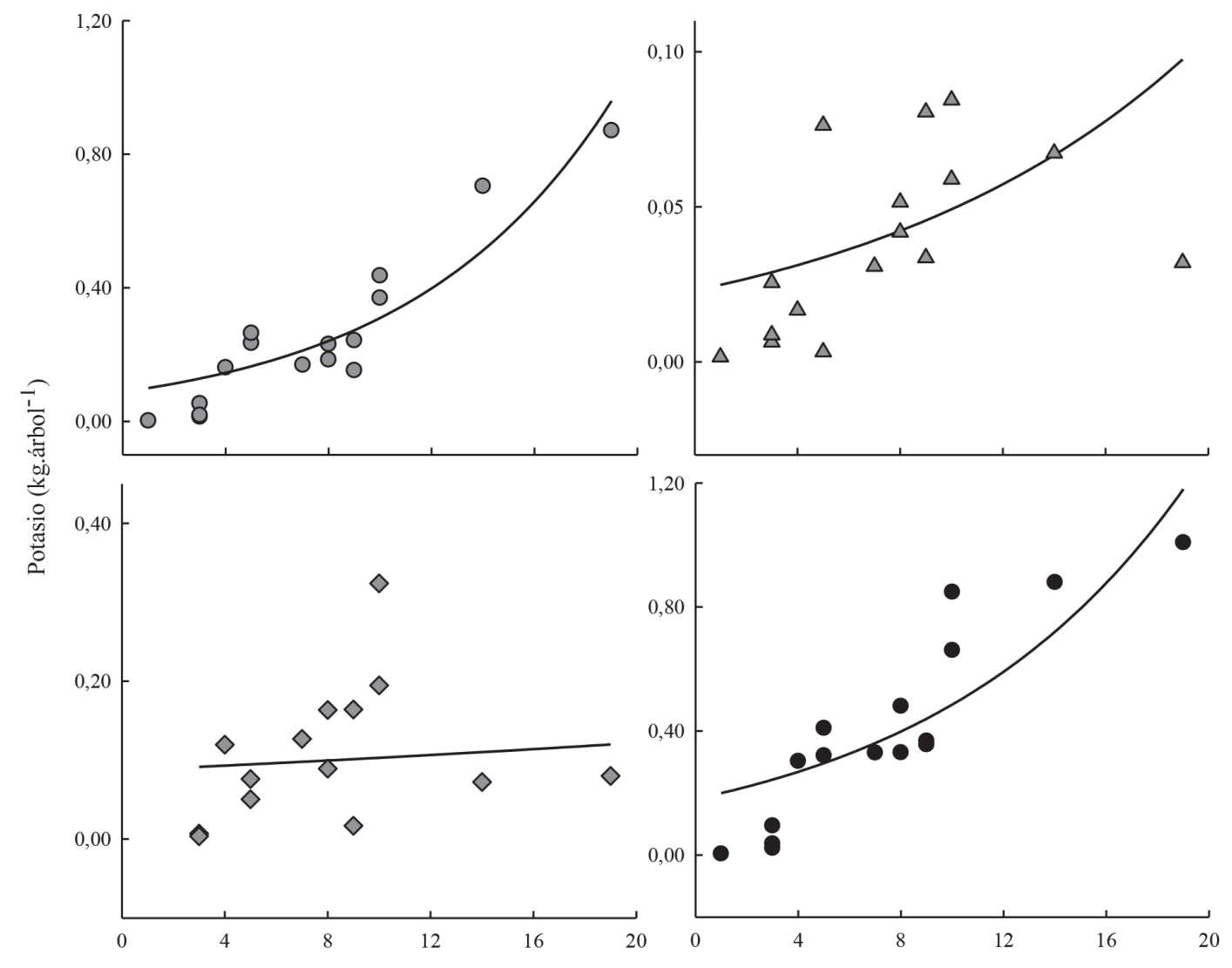

Edad (años)

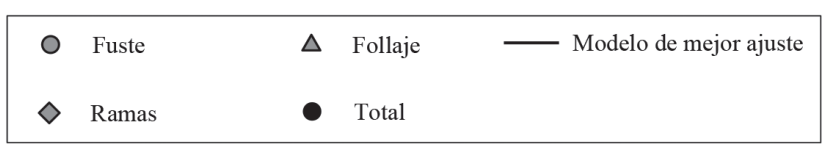

Fig. 9. Acumulación de potasio en función de la edad en los distintos componentes de la biomasa aérea de árboles de $C$. odorata del Trópico Muy Húmedo de Costa Rica. Los modelos de mejor ajuste se detallan en el Cuadro 3. 
De forma general, los modelos para medir la acumulación de los micro elementos en los componentes de la biomasa aérea durante los primeros 19 años de los árboles, presentaron una tendencia exponencial, así como p-valor inferiores a 0,05 en sus parámetros $(a)$ y $(b)$, a excepción del hierro y el aluminio (Cuadro 4).

Se observó que en el componente ramas los modelos presentaron valores de $\mathrm{R}^{2}$ inferiores a 0,02 . Destacan los microelementos cobre y boro, cuyos modelos para la acumulación total y en el fuste presentaron valores de $\mathrm{R}^{2}>0,65$. En el componente follaje, el comportamiento de estos mismos elementos fue similar a los componentes anteriormente mencionados, sin embargo, los valores de $\mathrm{R}^{2}$ fueron inferiores a 0,50 (Cuadro 4).

\section{DISCUSIÓN}

\section{Dinámica y acumulación de biomasa seca en los componentes de $C$. odorata}

En bosques tropicales la variabilidad en la tasa de crecimiento de las especies es producto de diversos factores como el suelo y la competencia por espacio y luz (Murillo 2008) que afectan la superficie fotosintética, y por ende, la capacidad del árbol para la fijación de $\mathrm{CO}_{2}$ (Kozlowski y Pallardy 1997), del efecto que tengan las plagas y malezas sobre los árboles y de la interacción de la especie con otros individuos. El crecimiento de las especies arbóreas del trópico húmedo aumenta entre 40 y $50 \%$ cuando se plantan con otras especies (Stanley y Montagnini 1999) y para $C$. odorata se ha demostrado que la especie crece hasta 5 veces más rápido cuando se planta en sistemas mixtos que como monocultivo, debido a una reducción del ataque de $H$. grandella con la edad de la plantación y a una menor densidad de individuos por parcela (Piotto et al. 2004).

La especie $C$. odorata tiende a formar copas estrechas a temprana edad, con una forma esférica formada por hojas compuestas en la parte superior y seguida por el desarrollo de ramificaciones (Hall y Ashton 2016). Al estudiar la relación $(\mathrm{H}+\mathrm{P}) /(\mathrm{F}+\mathrm{R})$ se nota que a edades tempranas
(0-3 años) la cantidad de tejido fotosintéticamente activo (hojas, raquis y pecíolos) es elevada en relación al tejido leñoso (ramas y fuste) y a partir del año 4 se reduce a valores entre 0,02 y 0,07 . Varios autores mencionan la disminución de la relación $\mathrm{H} / \mathrm{F}$ con la edad de los árboles en Betula spp., y Pinus taeda (Switzer et al. 1968), Pinus elliottii (Dickens et al. 2003), Tectona grandis (Portuguez 2012) y Alnus acuminata (Segura et al. 2005).

La curva de crecimiento de las especies arbóreas, incluyendo a $C$. odorata, tiende a ser sigmoidea (INAB 2015); sin embargo, en el presente trabajo se encuentran 3 etapas de crecimiento. En la primera etapa el crecimiento es lento, pese a que se ha reportado que el desarrollo inicial de $C$. odorata es ligeramente superior al de otras especies nativas (Hall et al. 2011). Además, cuando se planta en fincas de agricultores en vez de campos experimentales, su crecimiento se reduce, mostrando así, que las prácticas de manejo a nivel de finca son determinantes en parámetros de rentabilidad comercial (Hall et al. 2011).

De relevancia son la competencia con malezas por agua, nutrimentos y a menudo efectos alelopáticos (Andrés et al. 2011), por la intercepción de la luz (Delagrange et al. 2008) y la disponibilidad de nutrimentos (Pérez 2009). A partir de los 3 años (segunda etapa de crecimiento) los árboles inician la formación de ramas y el engrosamiento del fuste, este último es predominante en peso durante el resto de su vida. El comportamiento de la biomasa de ramas tiende al incremento, con una reducción marcada después de dicha edad, probablemente causada por competencia intraespecífica por luz y espacio para crecer (Ewell et al. 2014).

En la tercera etapa entre los 14 y 19 años ocurre una disminución de la biomasa de las hojas, pecíolos y ramas, la cual muestra que los árboles alcanzaron la máxima asimilación de carbono, probablemente debido al efecto de competencia intraespecífica y a una reducción del crecimiento causada por el ataque de $H$. grandella (Piotto et al. 2004, De la Rosa et al. 2011). En muchas especies arbóreas la llegada a 
la etapa madura está relacionada con la reducción de ramas y un balance en las reservas de energía, que en muchos casos puede ser motivo de la alta eficacia en la lucha contra enfermedades (Punches 2004). En esta etapa el crecimiento y la acumulación de biomasa en plantaciones de diferentes especies en el trópico están influenciados por la disponibilidad de agua (Lugo et al. 1988) y la densidad de la plantación (Foli et al. 2003).

El mejor modelo de regresión desarrollado en el presente trabajo para medir la biomasa aérea total $C$. odorata presentó un buen ajuste y significancia estadística, por lo que se considera satisfactorio en la determinación de biomasa en árboles de Cedrela en un rango de edades de 1 a 19 años. Saint-André et al. (2005) exponen que los modelos de biomasa pueden variar según la edad del rodal, no por la madurez del árbol, sino por un efecto combinado entre la edad y su condición dentro del rodal, ya que en plantaciones o en bosques no se puede dejar de lado el efecto que tienen los demás individuos. Otros modelos similares sobre acumulación de biomasa en especies tropicales son reportados para otras especies forestales en Costa Rica (Fonseca et al. 2009, Fernández et al. 2014) y en otros sitios se reportan comportamientos de crecimiento similares a los presentados en la Figura 2 (Vitousek y Sanford 1984, Yamada y Gholz 2002, Jiménez 2012).

Se generaron otros modelos similares (datos no presentados) para medir la biomasa seca del follaje y ramas, sin embargo, los parámetros de estos modelos no fueron estadísticamente significativos, posiblemente debido a la caducidad de la especie que causa el encontrar diferente cantidad de hojas en cierta época del año (Ewell et al. 2014).

\section{Concentración de nutrimentos en la biomasa aérea de $C$. odorata}

Para el nitrógeno, fósforo, potasio, calcio, magnesio, azufre se mencionan secuencias similares a las reportadas en el presente trabajo en varias especies forestales latifoliadas de Centro América y Costa Rica (Hunter y Stewart 1993, Alvarado et al. 2016), no así para la secuencia de los micro elementos, ya que se tiene poco conocimiento sobre ellos. Lo anteriormente mencionado es consecuencia de la absorción de nutrimentos desde los primeros meses de crecimiento cuando los árboles toman la mayoría de los elementos del suelo y luego las concentraciones dependen de su redistribución interna y de otros factores externos (Attiwill 1995). Posteriormente, la cantidad de nutrimentos asociada a los diferentes componentes depende de la biomasa aérea de la especie, edad, sitio donde se encuentre, la época del año y del tejido en que se tome la muestra, en el tanto en que estos factores afectan los procesos fisiológicos en los árboles (Salisbury y Ross 1992, Sampaio y Huber 1999, Marschner 2002).

Los valores de concentración de nitrógeno en el follaje de $C$. odorata obtenidos en el presente estudio fueron superiores al valor definido como de deficiencia por Dreschel y Zech (1991), lo que se debe probablemente al rol que juega el elemento en las células fisiológicamente activas de las hojas y tejidos meristemáticos (Kozlowki y Pallardy 1997). En el trópico la concentración de nitrógeno en la biomasa aérea puede disminuir con la precipitación pluvial anual de los sitios, como lo concluyen Santiago et al. (2004) en bosques de Panamá y tiende a disminuir con la edad (crecimiento) del árbol. Camacho et al. (2016) reportan comportamientos similares en la concentración de nitrógeno en los componentes de árboles de $V$.guatemalensis provenientes de plantaciones ubicadas en el trópico muy húmedo de Costa Rica. Al respecto, Fernández et al. (2013) consideran que la concentración de nitrógeno en los diferentes componentes de los árboles puede disminuir por 3 razones: 1) el efecto de dilución al aumentar la biomasa de acuerdo con la edad del árbol, por lo que tiende a producir tejidos estructurales, 2) la disminución de los requerimientos del árbol con la edad, por lo que se necesitará menos nitrógeno para mantener las tasas de crecimiento y 3) puede ocurrir una disminución en la cantidad de nitrógeno disponible en el suelo, lo que afectaría la absorción del nutrimento.

Otros factores que afectan la disponibilidad y absorción del nitrógeno son la 
descomposición (mineralización) de la materia orgánica y liberación del elemento, la fijación biológica del nitrógeno y otros factores ambientales como las adiciones por lluvia, la inmovilización del nitrógeno en la biomasa microbiana y su liberación posterior (Constantinides y Fownes 1994, Oehlund y Naesholm 2001). Como especie decidua se ha encontrado que los árboles de $C$. odorata pueden reciclar alrededor de 2 tercios del nitrógeno y un cuarto del fósforo absorbidos (Hiremath et al. 2002), sin contar la cantidad que puede quedar almacenada en sus raíces como órgano de almacenamiento (Millard 1995).

Los valores de concentración de fósforo obtenidos en el presente trabajo fueron los más bajos entre los macro nutrimentos y en el follaje los valores se encuentran en el rango de 0,10-0,19\% considerado como intermedio por Dreschel y Zech (1991) y Alvarado et al. (2016). Las bajas concentraciones de este nutrimento en los componentes de la biomasa aérea se consideran normales para especies forestales tropicales (Blanco et al.2005) y redundan en una eficiencia de uso elevada del elemento (Bündchen et al. 2013). En Panamá (Condit et al. 2013) y en la Amazonía de Brasil (Goodland y Pollard 1973, Harisadan 2000) se considera que el bajo contenido de fósforo en la biomasa aérea obedece a varios mecanismos de adaptación de las especies a la poca disponibilidad del elemento en suelos fuertemente meteorizados, al ser la producción de raíces proteoides asociadas con micorrizas el mecanismo que, además, puede afectar la distribución de la especie en el espacio y el tiempo (Watt y Evans 1999).

La concentración de potasio presentó comportamientos similares a los obtenidos para el nitrógeno en las hojas y el fuste. Este comportamiento se reporta en la literatura para otras especies forestales (Fernández et al. 2013, Camacho et al. 2016) y se relaciona a la gran movilidad del elemento, su función en la apertura de estomas, la asimilación de $\mathrm{CO}_{2}$ y la regulación de la tasa de transpiración (Binkley et al. 2002), por lo que se puede evidenciar una mayor concentración en las hojas que en el tronco conforme el árbol crece.
Las concentraciones de calcio en todos los componentes de la biomasa aérea de $C$. odorata se encontraron dentro del rango normal mencionado para especies latifoliadas de Costa Rica (Alvarado et al. 2016). Wessmann et al. (1989) concluyen que la concentración de calcio en árboles deficientes en el elemento puede ser menor en la parte alta de la copa, ya que este se debe transportar a largas distancias y existe una alta competencia en el flujo del xilema, comportamiento no observado en el presente estudio. $\mathrm{La}$ variación de la concentración foliar del azufre en varias especies forestales latifoliadas tropicales oscila entre 0,04 y $0,40 \%$ (Alvarado et al. 2016) y las concentraciones de $C$. odorata registradas en el presente estudio se ubican dentro del rango de las especies con valores más elevados $(0,15-$ 0,40\%) para Costa Rica (Alvarado 2012a).

Para la concentración de la mayoría de los micro elementos se encontró una tendencia de acuerdo con los componentes del árbol, a excepción del manganeso y el boro que se comportan al igual que los macro nutrimentos al existir una disminución de su concentración desde las hojas al tronco. La variación de la concentración de zinc en los diferentes componentes es bastante baja y permanece en alrededor de $30 \mathrm{ppm}$, valores que se encuentran dentro del rango considerado como normal en especies forestales (Boardman y McGuire (1990). Los valores de boro entre 100 y $600 \mathrm{mg} \cdot \mathrm{kg}^{-1}$ encontrados en los pecíolos pueden considerarse como muy altos, ya que a nivel foliar solamente pocas especies forestales tiene valores similares (Stone 1990, Lehto et al. 2010).

\section{Acumulación y distribución de nutrimentos en la biomasa aérea de $C$. odorata}

La acumulación de nitrógeno presentó una tendencia exponencial y es el elemento que más se acumula en los componentes de la biomasa del árbol, principalmente en el fuste. La absorción, transporte y uso dentro del árbol de compuestos nitrogenados específicos depende de la cantidad de nitrógeno disponible en el ecosistema, los sistemas absorción, la cantidad de transportadores y el metabolismo endógeno que permite la utilización 
de estos compuestos (Oehlund y Naesholm 2001). Normalmente durante las primeras etapas de crecimiento del árbol el nitrógeno en conjunto con el calcio, se acumulan en el follaje, para posteriormente acumularse en el fuste durante el crecimiento activo del árbol (Nwoboshi 1984, Ola-Adams 1993). Este comportamiento se reporta en especies nativas y exóticas en regiones de Costa Rica (Segura et al. 2005, Arias et al. 2011, Fernández et al. 2013, Camacho et al. 2016).

El calcio es uno de los elementos más importantes en la formación de la madera; a nivel celular este elemento tiene funciones como mensajero en diferentes procesos fisiológicos en las membranas de paredes celulares de los troncos de los árboles, donde tiene un papel fundamental en su estabilidad y en la integridad estructural de la célula (Lautner y Fromm 2010). Pese a que Wessmann et al. (1989) exponen que las concentraciones de calcio tienden a ser menores en la sección más alta de la copa de los árboles, en el presente trabajo no se observó dicho comportamiento.

Se registraron valores de fósforo acumulado en el fuste similares a los reportados por Camacho et al. (2017) para árboles de V. guatemalensis en condiciones agroecológicas similares a las reportadas para las plantaciones de Cedrela estudiadas en el presente trabajo. Este comportamiento podría deberse a mecanismos internos que propician un bajo uso de este macro nutrimento de acuerdo con lo reportado por varios autores para especies adaptadas a condiciones de acidez de suelo (Rao et al. 1999, Watanabe y Osaki 2002, Kochian et al. 2004). La absorción de fósforo del suelo ocurre principalmente en los primeros $10 \mathrm{~cm}$ del mismo, donde la concentración de raíces finas es más profusa y el elemento más abundante, debido a una mayor actividad de enzimas fosfatasas que libera el fósforo necesario para el desarrollo del árbol (Imai et al. 2012).

Los modelos generados para medir la acumulación de nutrimentos en la biomasa de follaje ramas y fuste permiten calcular la extracción de nutrimentos del ecosistema y, por ende, la necesidad de posibles adiciones al sistema para reponer lo extraído como material removido (Palm 1995, Yamada et al. 2004). Para mantener la productividad de los sitios a largo plazo es importante minimizar la exportación de biomasa, así como dar un manejo adecuado a los desechos de cosecha y reponer los nutrimentos extraídos debido a la cosecha (Thiers et al. 2007). Estos resultados ponen en evidencia la importancia que tienen las partes no comerciales del árbol de $C$. odorata desde el punto de vista de nutricional, ya que poseen gran cantidad de nutrimentos que se pueden incorporar al sistema una vez que estos restos se descomponen.

\section{CONCLUSIONES}

De forma general, los valores de concentración de macro nutrimentos y micro elementos en los componentes de la biomasa aérea en árboles de $C$. odorata tienden a disminuir con la edad y de forma diferenciada en los distintos componentes de la biomasa aérea. Destacan los modelos de regresión lineal desarrollados para evaluar este comportamiento en el fuste, los cuales presentaron tendencia a la disminución de la concentración nitrógeno, potasio y magnesio con el incremento de la edad. Estos comportamientos obedecen a mecanismos fisiológicos que permiten a las especies el desarrollo en ambientes con condiciones de suelo distróficas.

Se encontró valores máximos de acumulación de nitrógeno, calcio y potasio en el fuste, este componente es el de mayor aporte en la acumulación total de nutrimentos en la biomasa aérea. Los modelos de regresión desarrollados para medir estos valores de acumulación presentaron un muy buen ajuste, principalmente en la acumulación total y en el fuste, lo cual permite el cálculo de la cantidad de nutrimentos que se exportan del sitio de plantación, así como la estimación de índices de estabilidad de sitio con miras a futuras rotaciones.

Los modelos desarrollados para medir la acumulación de nutrimentos en los componentes ramas y follaje permiten medir posibles cantidades de nutrimentos que permanecen en el sitio de 
plantación como parte del reciclaje de nutrimentos, generando información útil en la gestión de la nutrición mineral de la especie, con miras a producciones sostenibles.

Se recomienda continuar con la investigación de esta especie, en específico replicar este tipo de estudios en plantaciones de $C$. odorata, cuyas condiciones edáficas y agroecológicas con el objetivo de mejorar el conocimiento de la dinámica de los nutrimentos en esta especie bajo distintos escenarios, considerada de gran valor económico.

\section{AGRADECIMIENTOS}

Por su colaboración en el proceso de colectar y analizar el material vegetal del presente trabajo les quedamos agradecidos al personal de campo de CoopeAgri R.L. y de la EARTH y al personal de los Laboratorios de Suelos y Foliares del CIA. Se extiende un agradecimiento especial al Dr. Raúl Jaramillo del IPNI y a M.Sc. Floria Bertsch del CIA/UCR por facilitar el financiamiento parcial del presente trabajo.

\section{LITERATURA CITADA}

Alvarado, A. 2012a. Nutrición y fertilización de Cedrela odorata. In Alvarado A, Raigosa J (eds.). Nutrición y fertilización forestal en regiones tropicales. San José, Costa Rica. p. 213-219.

Alvarado, A. 2012b. Reciclaje de nutrimentos en plantaciones $\mathrm{y}$ bosques tropicales. In Alvarado A, Raigosa J (eds.). Nutrición y fertilización forestal en regiones tropicales. San José, Costa Rica. ACCS. p. 51-96.

Alvarado, A; Camacho, ME; Raigosa, J; Villalobos, JE; Mora, A; Fernández, J; Murillo, R; Ávila, CA. 2016. Interpretación del análisis foliar de especies forestales latifoliadas del trópico americano. In XXI Congreso Latinoamericano de la Ciencia del Suelo. Quito, Ecuador. 44 p.

Andrés, P; Salgado, C; Espeleta, JM. 2011. Optimizing nursery and plantation methods to grow Cedrela odorata seedlings in tropical dry agroecosystems. Agroforest Systems 83:225-234.

Attiwill, P. 1995. Nutrient cycling in plants. In W.A. Nierenberg (ed.). Encyclopedia of Environmental Biology, Academic Press, San Diego 2:624-629.

Avellán MJ, Murillo R, Alvarado A, Ávila C. 2015. Variación del contenido foliar de nutrimentos de Gmelina arborea en los cantones de Osa, Golfito y Corredores, Costa Rica. Revista de Ciencias Ambientales (Trop. J. Environ. Sci.) 49(1):1-15.

Belanger, RP; Briscoe, CB. 1963. Effects of irrigating tree seedlings with a nutrient solution. Caribbean Forester 24(2):87-90.

Binkley, D; Stape, J; Ryan, M; Barnard, H; Fownes, J. 2002. Age-related decline in forest ecosystem growth: an individual-tree, stand-structure hypothesis Ecosystems 5:58-67.

Blanco, MA; Carpio, IM; Muñoz, F de M. 2005. Fichas técnicas de veinte especies maderables de importancia comercial en Costa Rica. San José, Costa Rica, Editorial de la Universidad de Costa Rica. 102 p.

Boardman, R; McGuire, DO. 1990. The role of zinc in forestry. I. Zinc in forest environments, ecosystems and tree nutrition. Forest Ecology and Management 37(1-3):167-205.

Bolaños, R; Watson, V; Tosi J. 2005. Mapa ecológico de Costa Rica (Zonas de Vida), según el sistema de clasificación de zonas de vida del mundo de L.R. Holdridge, Escala 1:750 000. San José, Costa Rica, Centro Científico Tropical.

Büendchen, M; Boeger, MRT; Reissmann, CB; da Silva, SLC 2013. Nutritional status and nutrient use efficiency in tree species of subtropical forest in southern Brazil. Scientia Forestalis 41(98):227-236.

Camacho, ME; Alvarado, A; Fernández, J. 2016. Vochysia guatemalensis Donn. Smith, an alternative species for reforestation on acid tropical soils. New Forests 47(4):497-512.

Camacho, ME; Alvarado, A; Fernández, J. 2017. Accumulation and export of nutrients in harvested wood of Vochysia guatemalensis in small-scale forest plantations. Journal of Tropical Forest Science 29(1):105-113.

Castaing, A. 1982. Algunos factores edáficos y dasométricos relacionados con el crecimiento y comportamiento de Cedrela odorata L. Tesis M.Sc. Programa Recursos Naturales UCR/CATIE. Turrialba, Costa Rica. 123 p.

Cavers, S; C Navarro, C; Lowe, AJ. 2003. Chloroplast DNA phylogeography reveals colonization history of a Neotropical tree, Cedrela odorata L., in Mesoamerica. Molecular Ecology 12(6):1451-1460.

Condit, R; Engelbrecht, BJM; Delicia-Pino, D; Pérez, R; Turner, BL. 2013. Species distributions in response to individual soil nutrients and seasonal drought across a community of tropical trees. Proceedings of the National Academy of Sciences 110(13):5064-5068.

Constantinides, M; Fownes J. 1994. Nitrogen mineralization from leaves and litter of tropical plants: relationship to nitrogen, lignin and soluble polyphenol concentrations. Soil Biology and Biochemistry 26(1):49-55. 
Delagrange, S; Potvin, C; Messier, C; Coll, L. 2008. Linking multiple-level tree traits with biomass accumulation in native tree species used for reforestation in Panama. Trees 22:337-349.

De la Rosa, V; Díaz, M; Contreras, G; Jiménez, C; Edzná, C; Mocochá, C. 2011. Effects of environmental factors on the incidence of Hypsipyla grandella Zeller in plantations of Cedrela odorata L. In VI Reunión Nacional de Innovación Forestal. León, Guanajuato, MX. 85 p.

Dickens, DE; Moorhead, DJ; McElvany, BM. 2003. Georgia, phosphorus fertilization at establishment in loblolly and slash pine stands on Atlantic and Gulf Coastal Plain sites. Better Crops 87(1):12-15.

Di Rienzo, JA; Casanoves, F; Balzarini, MG; Gonzalez, L; Tablada, M; Robledo, CW. 2012. InfoStat versión 2012. (en línea, programa informático). Grupo InfoStat, FCA, Universidad Nacional de Córdoba, Argentina. 5 nov. 2014. Disponible en http://www. infostat.com.ar

Drechsel, P; Zech, W. 1991. Foliar nutrient levels of broadleaved tropical trees: A tabular review. Plant and Soil 131:29-46.

Drechsel, P; Zech, W. 1993. Mineral nutrition of tropical trees. In Pancel L. (eds.). Tropical Forestry Handbook. Berlin, Alemania, Springer Verlag. p. 515-567.

Ewell, JJ; Mazzarino, MJ; Celis, G. 2014. Soil changes in model tropical ecosystems: effects of stand longevity outweigh plan diversity and tree species identity in a fertile volcanic soil. Ecosystems 17:820-836.

Fernández, J; Murillo, R; Portuguez, E; Fallas, JL; Rios, V; Kottman, F; Verjans, JM; Mata, R; Alvarado, A. 2013. Nutrient concentration age dynamics of teak (Tectona grandis L f) plantations in Central America. Forest Systems 22(1):123-133.

Fernández, J; Murillo, R; Portuguez, E; Fallas, JL; Rios, V; Kottman, F; Verjans, JM; Mata, R; Alvarado, A. 2014. Nutrient accumulation and export in teak (Tectona grandis L.f.) plantations of Central America. Doi: 10.3832/ifor1089-007 iForest-Biogeosciences and Forestry 8:33-44.

Foli, E; Alder, D; Miller, H; Swaine, H. 2003. Modeling growing space requirements for some tropical forest tree species. Forest Ecology and Management 173:79-88.

Fonseca, W; Alice, F; Rey, JM. 2009. Modelos para estimar la biomasa de especies nativas en plantaciones y bosques secundarios en la zona Caribe de Costa Rica. Bosque 30(1):36-47.

Goodland, R; Pollard, R. 1973. The Brazilian cerrado vegetation: a fertility gradient. Journal of Ecology 61(1):219-224 .

Guevara, G. 1988. Experiencias colombianas con cedro (Cedrela odorata L.). Corporación Nacional de Investigación y Fomento Forestal. Bogotá, Colombia. 86 p. Serie de Documentación, N ${ }^{\circ} .12$.
Hall, JS; Ashton, MS. 2016. Guía de crecimiento y sobrevivencia temprana de 64 especies de árboles nativos de Panamá y el neotrópico. Instituto Smithsoniano de Investigaciones Tropicales. Balboa, Panamá. 171 p.

Hall, JS; Love, BE; Garen, EJ; Slusser, L; Saltonstall, K; Mathias, S; van Breugel, M; Ibarra, D; Bork, EW; Spaner, D; Wishnie, MH; Ashton, MS. 2011. Tree plantation on farm: evaluating growth and potential for success. Forest Ecology and Management 261:1675-1683.

Haridasan, M. 2000. Nutrição mineral de plantas nativas do Cerrado. Revista Brasileira Fisiología Vegetal 12(1):54-64.

Hiremath, A; Ewel, J; Cole, T. 2002. Nutrient use efficiency in three fast-growing tropical trees. Forest Science 48(4):662-672.

Hunter, IR; Stewart, MS. 1993. Foliar nutrient and nutrient content of Central American multipurpose tree species growing at Comayagua, Honduras. Commonwealth Forestry Review 72(3):193-197.

Imai, N; Kitayama, K; Titin, J. 2012. Effects of logging on phosphorus pools in a tropical rainforest of Borneo. Journal of Tropical Forest Science 24(1):5-17.

INAB (Instituto Nacional de Bosques, Guatemala, Ministerio de Agricultura, Ganadería y Alimentación). 2015. Dinámica de crecimiento y productividad de 28 especies forestales en plantaciones en Guatemala, Serie Técnica No. DT-002(2015). Guatemala. 212 p.

Jiménez, N. 2012. Producción de madera y almacenamiento de carbón en cafetales con cedro (Cedrela odorata) y caoba (Swietenia macrophylla) en Honduras. Tesis M.Sc. Agroforestería Tropical. CATIE. Costa Rica. 91 p.

Kalra, Y. 1998. Handbook of reference methods for plant analysis. Soil and Plant Analysis Council, Inc. Boca Raton, FLA, CRC Press. 300 p.

Kochian, LV; Hoekenga, OA; Piñeros, MA. 2004. How do crop plants tolerate acid soils? Mechanisms of aluminum tolerance and phosphorous efficiency. Annual Review Plant Biology 55:459-493.

Kozlowski, T; Pallardy, S. 1997. Physiology of woody plants. Tercera edición. Academic Press. San Diego, California. USA. 811 p.

Lautner, S; Fromm, J. 2010. Calcium-dependent physiological processes in trees. Plant Biology 12:268-274.

Lehto, T; Ruuhola, T; Dell, B. 2010. Boron in forest trees and forest ecosystems. Forest Ecology and Management 260:2053-2069.

Lugo, A; Brown, S; Chapman, J. 1988. An analytical review of production rates and stemwood biomass of tropical forest plantations. Forest Ecology and Management 23:179-200.

Marschner, H. 2002. Mineral nutrition of higher plants. $2^{\mathrm{a}}$. Ed. Academic Press. 4-16 p. 
Mateo, JJ; Bonifacio, R; Pérez, SR; Capulín, J; Mohedano, L. 2011. Producción de Cedrela odorata L. en aserrín crudo con diferentes dosis de fertilización, en Tecpán de Galeana, Guerrero. Ra Xinhai 7(2):195-204.

Millard, P. 1995. Internal cycling of nitrogen in trees. Acta Horticulturae 386:3-14.

Murillo, Y. 2008. Determinación de aclareo en plantaciones de cedro (Cedrela odorata L.) en el estado de Tabasco. Tesis M.Sc. Producción Agroalimentaria en el Trópico, Campus Tabasco. Colegio de Posgraduados. Tabasco, México. 88 p.

Murphy, J; Riley, JP. 1962. A modified single solution for determination of phosphate in nature waters. Anal. Chem. Acta 27:31-36.

Nwoboshi, LC. 1984. Growth and nutrient requirements in a teak plantation age series in Nigeria. II. Nutrient accumulation and minimum annual requirements. Forest Science 30(1):35-40.

Oehlund, J; Naesholm, T. 2001. Growth of conifer seedlings on organic and inorganic nitrogen sources. Tree Physiology 21:1319-1326.

Ola-Adams, BA. 1993. Effects of spacing on biomass distribution and nutrient content of Tectona grandis Linn. f. (teak) and Terminalia superba Engl. and Diels. (afara) in south-western Nigeria. Forest Ecology Management 58:299-319.

Palm, C. 1995. Contribution of agroforestry trees to nutrient requirements of intercropped plants. Agroforestry Systems 30:105-124.

Paniagua, A. 2005. Respuesta de Cedrela odorata a dosis crecientes de carbonato de calcio y $\mathrm{P}_{2} \mathrm{O}_{5}$ en un Fluventic Dystropept en invernadero. In Memorias Primer Congreso sobre Suelos Forestales. Universidad Nacional, INISEFOR. Heredia, Costa Rica. $11 \mathrm{p}$.

Pérez, G. 2009. Caracterización y determinación del índice de sitio en plantaciones de Swetenia macrophylla en Tabasco, México. Tesis M.Sc. Chapingo, Campus de Tabasco, México. 79 p.

Piotto, D; Víquez, E; Montagnini, F; Kanninen, M. 2004. Pure and mixed plantations with native species of the dry tropics of Costa Rica: a comparison of growth and productivity. Forest Ecology and Management 190(2-3):359-372.

Portuguez, EM. 2012. Estimación de la extracción de nutrimentos por parte aérea en plantaciones de teca (Tectona grandis Linn. f.) de las empresas Panamerican Woods y C\&M Investment Group Ltda. en la península de Nicoya, Guanacaste, Costa Rica. B.S. Tesis Lic. San José, Costa Rica. Universidad de Costa Rica. 92 p.

Punches, J. 2004. Tree growth, forest management and their implications for wood quality. Oregon State University. Pacific Northwest Extension Publishing 576:2-8.
Rao, IM; Friesen, DK; Osaki, M. 1999. Plant adaptation to phosphorus-limited tropical soils In Pessarakli M (ed.) Handbook of Plant and Crop Stress. $2^{\mathrm{a}}$ edición. Marcel Deker Inc, New York. p. 61-95.

Rodríguez, M; López, R; Martínez, R; Hernández, G; Sarmiento, O. 2015. Respuesta del cedro (Cedrela odorata L.) a diferentes dosis de riego. Revista Ciencias Técnicas Agropecuarias 24(4):12-15.

Saint-André, L; M’Bou, AT; Mabiala, A; Mouvondy, W; Jourdan, C; Roupsard, O; Deleporte, P; Hamel, O; Nouvellon, Y. 2005. Age-related equations for above- and below-ground biomass of a Eucalyptus hybrid in Congo. Forest Ecology and Management 205(1-3):199-214.

Salisbury, F; Ross, C. 1992. Fisiología vegetal. Ed. Iberoamérica. p. 149-151.

Sampaio, A; Huber, D. 1999. Concentração e distribuição de nutrimentos nas folhas de especies florestais na Amazonia Ocidental. Floresta e Ambiente 6(1):127-137.

Santiago, L; Kitajima, K; Wright, S; Mulkey, S. 2004. Coordinated changes in photosynthesis, water relations and leaf nutritional traits of canopy trees along a precipitation gradient in lowland tropical forest. Oecologia 139(4):495-502.

Segura, M; Castillo, A; Alvarado, A; Blanco, F. 2005. Extracción de nutrimentos en plantaciones de jaúl (Alnus acuminata) en la cuenca del río Virilla, Costa Rica. Agronomía Costarricense 29(2):109-120.

Segura, M; Castillo, A; Alvarado, A; Blanco, F. 2006 Variación del contenido foliar de nutrimentos de Alnus acuminata. Agronomía Costarricense 30(1):53-63.

Stanley, W; Montagnini, F. 1999. Biomass and nutrient accumulation in pure and mixed plantations of indigenous tree species grown on poor soils in the humid tropics of Costa Rica. Forest Ecology and Management 113(1):91-103.

Stone, EL. 1990. Boron deficiency and excess in forest trees: a review. Forest Ecology and Management 37(13):49-75.

Switzer, GL; Nelson, LE; Smith, WH. 1968. The mineral cycle in forest stands. In Tennessee Valley Authority (eds.). Forest Fertilization theory and practice. National Fertilizer Development Center. Muscle Shoals, Alabama. p. 1-9.

Systat Software, Inc. 2008. SigmaPlot for Windows, version 11.0. Systat Software, Inc., San Jose, CA.

Thiers, O; Gerding, V; Schllater, J. 2007. Exportación de nitrógeno y calcio mediante raleo en un rodal de Eucalyptus nitens de 5 años de edad, Chile. Bosque 28(3):256-262.

Timyan, J. 1996. BWA YO: Important tree of Haiti. SECID. Washington. USA. p. 63-70. 
Vitousek, PM; Sandfor, RL. 1984. Nutrient cycling in moist tropical forest. Annual Review of Ecology and Systematics 17:137-167.

Watanabe, T; Osaki, M. 2002. Mechanisms of adaptation to high aluminum condition in native plant species growing in acid soils: a review. Communications in Soil Science and Plant Analysis 33:1247-1260.

Watt, M; Evans, JR. 1999. Proteoid roots: Physiology and development. Plant Physiology 121:317-323.

Webb, M; Reddell, P; Hambleton, A; Robson, K. 2000. Growth response of four tropical plantation timber species to increasing phosphorus supply and assessment of phosphorus requirements using foliar analysis. New Forests 20(2):193-211.

Webb, MJ; Reddell, P; Grundon, NJ. 2001a. A visual guide to nutritional disorders of tropical timber species: Swietenia macrophylla and Cedrela odorata. Camberra, Australia. 178 p. ACIAR Monograph, No. 61 .
Webb, MJ; Reddell, P; Nath, S; Srivastava, RJ. 2001b. Determining $\mathrm{P}$ an $\mathrm{N}$ status of a tropical timber species (teak): assessment of a quick chemical test and root phosphatase assay. In Horst WJ (ed). XIV International Plant Nutrition, Plant nutrition, food security - Food security and sustainability of agroecosystems. 2001. p. 706-707.

Wessmann, C; Aber, J; Peterson, D. 1989. An evaluation of imaging spectrometry for estimating forest canopy chemistry. International Journal of Remote Sensing 10:1293-1316.

Yamada, M; Gholz, H. 2002. Growth and yield of some indigenous trees in an Amazonian agroforestry system: rural-history-based analysis. Agroforestry Systems 55(1):17-26.

Yamada, M; Toma, T; M Hiratsuka, Y Morikawa, Y. 2004. Biomass and potential nutrient removal by harvesting in short-rotation plantations. In Nambiar, E; Ranger, J; Tiarks, A (eds.). Site Management and Productivity in Tropical Plantation Forests. CIFOR 14 p. 